Discussion Paper Series A No.715

\title{
Nonconvex Bargaining Problems: Some Recent Developments
}

Yongsheng Xu

(Department of Economics, Andrew Young School of Policy Studies,

Georgia State University)

and

Naoki Yoshihara

(Department of Economics, University of Massachusetts Amherst)

October 2020

Institute of Economic Research

Hitotsubashi University

Kunitachi, Tokyo, 186-8603 Japan 


\title{
Nonconvex bargaining problems: some recent developments
}

\author{
Yongsheng $\mathrm{Xu}$ \\ Department of Economics, Andrew Young School of Policy Studies \\ Georgia State University, Atlanta, GA 30303 \\ E-mail: yxu3@gsu.edu \\ Naoki Yoshihara \\ Department of Economics, University of Massachusetts Amherst \\ Amherst, MA 01002 \\ E-mail: nyoshihara@econs.umass.edu
}

May 14, 2020

\begin{abstract}
This paper presents a survey of the recent theoretical results on solutions to nonconvex bargaining problems. We discuss rationalizability of solutions to nonconvex bargaining problems, axiomatic characterizations of the symmetric and asymmetric Nash solution, the Kalai-Smorodinsky solution, and the egalitarian solution to nonconvex bargaining problems, and applications in the context of resource allocation and of bargaining over opportunity sets.
\end{abstract}

Keywords: nonconvex bargaining problem, Nash solution, equitable Nash solution, Kalai-Smorodinsky solution, Egalitarian solution, resource allocation problem

JEL Classification Numbers: C71, C78, D63, D71 


\section{Introduction}

An $n$-person bargaining problem is a pair $(S, d)$ where $S$ is a non-empty subset of the $n$ dimensional real space, $\mathbb{R}^{n}$, and $d$ is an element of $S$. The set $S$ is typically interpreted as the attainable set of utility allocations each of which can be obtained by $n$ players through their joint action. The element $d$ in $S$ is interpreted as the threat or disagreement point: if the players fail to reach an agreement, then the bargaining problem $(S, d)$ will be settled at $d$. Depending on specific situations, bargaining problems can be different. Collect all bargaining problems in a set $\Sigma$. A bargaining solution defined on $\Sigma$ picks up an element in a bargaining set $S$ for each bargaining problem $(S, d)$ contained in $\Sigma$ : the picked up element by the bargaining solution for a problem is regarded as the settlement point by the players for that problem. There can be different bargaining solutions satisfying various properties like efficiency, symmetry, etc. In Nash's (1950) pioneering work on bargaining, he takes an axiomatic approach to study what is now called the Nash solution: he shows that there is a unique solution satisfying a list of reasonable properties (axioms) and thus obtains an axiomatic characterization of the Nash solution. Several other solutions including the Kalai-Smorodinsky solution (Kalai and Smorodinsky (1975)) and egalitarian solution (Kalai (1977)) have been axiomatically studied in the literature.

The collection of bargaining problems studied by Nash (1950) and subsequently by others is such that, for each bargaining problem $(S, d)$, the bargaining set $S$ is convex. However, it has been recognized that the attainable set of utility allocations arising from many economic contexts may not be convex. One of the earliest researchers who recognizes such nonconvexity in a bargaining problem is Bishop (1960). Bishop (1960) studies the behaviors of duopoly and shows that the bargaining set for a duopoly bargaining problem can be non-convex when the two firms face conventional demands but have unequal marginal costs. He further cautions that, if side payments are prohibited, then the Nash solution to convex bargaining problems is not applicable in this context.

Non-convexity of the attainable set of utility allocations can be removed if it is assumed that players are expected utility maximizers and if the use of lotteries is admitted. In recent decades, these assumptions have been under increasing scrutiny and many researchers have questioned their validity. Non-convexity of bargaining sets thus invites questions about the model and analysis of (classical) convex bargaining problems: How can the classical model and analysis developed by Nash (1950) be extended to bargaining problems with nonconvex bargaining sets? The recent literature on bargaining problems has examined this question and has provided a foundation for applying the theories of nonconvex bargaining problems thus developed to several economic contexts in which nonconvexity of bargaining sets arises naturally.

The purpose of this paper is two-fold. First, we review some major theoretical results in the recent literature on $n$-person non-convex bargaining problems. Secondly, we use two resource allocation problems that give rise to nonconvex bargaining sets to illustrate potential applications of the theories of nonconvex bargaining problems. In our review of the related theoretical literature, we focus on a collection of bargaining problems $(S, d)$ in which: (i) the threat or disagreement point $d=\left(d_{1}, \cdots, d_{n}\right)$ is fixed, (ii) any bargaining problem $(S, d)$ is such that, for any point $s=\left(s_{1}, \cdots, s_{n}\right)$ in $S, s_{1} \geq d_{1}, \cdots, s_{n} \geq d_{n}$, and (iii) each player has a cardinal utility function. With these assumptions, we consider normalized bargaining 
problems $(S, d)$ where $d=(0, \cdots, 0), S \subseteq \mathbb{R}_{+}^{n}\left(\mathbb{R}_{+}^{n}\right.$ being the $n$-fold Cartesian product of the set of non-negative real numbers) is compact and comprehensive, but not necessarily convex. See Section 2 for formal definitions.

As we noted earlier, the theoretical study of $n$-person bargaining problems is typically axiomatic. We shall follow the axiomatic approach in our review of major theoretical results on $n$-person non-convex bargaining problems. Unlike $n$-person convex bargaining problems where a bargaining solution picks up a unique point in the bargaining set $S$, a solution in $n$-person nonconvex bargaining problems can be multi-valued. For example, there can be several points in a nonconvex bargaining set that maximize the Nash product. Depending on how a Nash solution is defined for such bargaining problems, a Nash solution can be set-valued or single-valued. We shall discuss both set-valued and single-valued Nash solutions for nonconvex bargaining problems. In addition, we shall also discuss some other prominent solutions (Kalai-Smorodinsky, Egalitarian) to bargaining problems and the issue of rationalizability of bargaining solutions axiomatically.

From our discussions, we will see that many of the axioms used for studying solutions to convex bargaining problems continue to play a major role in characterizing solutions to nonconvex bargaining problems. These axioms include Efficiency, Weak Efficiency, Scale Invariance, Individual Rationality, Contraction Independence (Nash's IIA) (see Section 3 for formal definitions). We will also see that, for the class of nonconvex bargaining problems reviewed in this paper, the characterization results of the Kalai-Smorodinsky solution and the Egalitarian solution can be regarded as straightforward extensions of their counterparts for the class of convex bargaining problems. See Sections 6 and 7. On the other hand, depending on the nature of nonconvex bargaining problems, one would need to face the challenge of defining a Kalai-Smorodinsky solution or an egalitarian solution appropriately: when a nonconvex bargaining problem contains only a finite number of points, the standard Kalai-Smorodinsky solution and the standard Egalitarian solution may not be well-defined (see Section 2)!

In the meantime, there are new axioms that are specific for studying solutions to nonconvex bargaining problems. Consequently, solutions to nonconvex bargaining problems exhibit some common features to their counterparts to convex bargaining problems and, simultaneously, have certain new features that are unique to them so that new insights can be derived from axiomatic studies. For example, consider first Nash's original symmetry axiom (for convex bargaining problems), which requires that, for any convex bargaining problem $(S, d)$ in which $S$ is symmetric with respect to $d$, the solution point $s^{*} \in S$ should be such that $s_{i}^{*}-d_{i}=s_{j}^{*}-d_{j}$ for all $i, j=1, \cdots, n$. Note that Nash's symmetry property captures an anonymity property and an equity property implicitly embedded in it, and in Nash's framework, these two properties cannot be disentangled. However, in nonconvex bargaining problems, we can extend Nash's symmetry axiom to two versions (S) and (SS) (see Section 3 for formal definitions and discussions): Consider a bargaining problem $(A, d)$ with $d=0$ such that $A$ is symmetric; then, the axiom (S) requires a solution be anonymous-if $a \in A$ is a solution candidate, so is any permutation of $a$, while (SS) requires a solution be equitable-any solution candidate $a \in A$ must be such that $a_{1}=\cdots=a_{n}$. Consequently, for nonconvex bargaining problems, we can make a distinction between Anonymity and Equity requirements simultaneously embedded in Nash's original symmetry axiom. An implication of making this distinction is that the presence of multi-valued solution to nonconvex bargaining problems 
may allow the existence of anonymous but inequitable solutions. Indeed, from our characterization and refinement results of the Nash solution for nonconvex problems (see Section 5), we see clearly the possibility of having anonymous and inequitable solutions to nonconvex bargaining problems. This feature and insight of the Nash solution is inherent to nonconvex bargaining problems. Second, consider the issue of rationalizability of a bargaining solution. For nonconvex bargaining problems, a solution may be set-valued. Consequently, we need certain 'expansion' type properties developed in rational choice theory in addition to variants of Nash's IIA to study rationalizability of a solution to nonconvex bargaining problems. Our results on rationalizability of a solution in Section 4 show that, (1) once we take care of expansion type axioms, the results obtained in the context of convex bargaining problems can be extended to the context of nonconvex bargaining problems, and (2) the rationality requirements in rationalizing bargaining solutions to nonconvex bargaining problems are less stringent than those in rational choice theory.

The Nash and other prominent solutions to convex bargaining problems have been fruitfully applied in many settings in economics. Given the recent development of theories of nonconvex bargaining problems, one would naturally wonder how and to what extent the developed theory can be applied in such settings. For this purpose, we shall review two applications of theories of nonconvex bargaining problem to economics where nonconvex bargaining problems arise naturally: one instance is when individuals are not characterized by their utilities but by their functionings and capability sets à la Sen (1985), and the other is when discussing distributive justice in a standard problem of resource allocations in economic environments. We will see the reviewed theories of nonconvex bargaining problems can be readily applied to those economic settings (see Section 8 for details).

The paper is organized as follows. Section 2 presents notation and definitions needed for our discussion. Section 3 introduces several basic axioms used for various axiomatic characterizations of the well-known solutions. In Section 4, we discuss rationalizability of bargaining solutions to nonconvex bargaining problems. Section 5 is devoted to the discussion of the Nash solution: characterization, refinement, and extension. Sections 6 and 7 discuss, respectively, characterizations of the Kalai-Smorodinsky solution and the Egalitarian solution. In Section 8, we provide two economic applications to illustrate how nonconvex bargaining theories can be applied to resource allocation problems and to the problem of bargaining over opportunity sets. Section 9 contains a brief conclusion.

\section{Notation and definitions}

$\mathbb{R}_{+}$is the set of all non-negative real numbers and $\mathbb{R}_{++}$is the set of all positive numbers. $\mathbb{R}_{+}^{n}$ (resp. $\mathbb{R}_{++}^{n}$ ) is the $n$-fold Cartesian product of $\mathbb{R}_{+}$(resp. $\mathbb{R}_{++}$). For any $x, y \in \mathbb{R}_{+}^{n}$, we write $x>y$ to mean $\left[x_{i} \geq y_{i}\right.$ for all $i \in N$ and $\left.x \neq y\right]$, and $x \gg y$ to mean $\left[x_{i}>y_{i}\right.$ for all $\left.i \in N\right]$. For any $x \in \mathbb{R}_{+}^{n}$ and any non-negative number $t$, we write $z=\left(t ; \mathbf{x}_{-i}\right) \in \mathbb{R}_{+}^{n}$ to mean that $z_{i}=t$ and $z_{j}=x_{j}$ for all $j \in N \backslash\{i\}$. For any $A \subseteq \mathbb{R}_{+}^{n}$ and any $\alpha \in \mathbb{R}_{+}^{n}, \alpha A$ denotes the set $\left\{\left(\alpha_{1} a_{1}, \cdots, \alpha_{i} a_{i}, \cdots, \alpha_{n} a_{n}\right) \mid a \in A\right\}$. For any subset $A \subseteq \mathbb{R}_{+}^{n}, A$ is said to be (i) non-trivial if there exists $a \in A$ such that $a \gg \mathbf{0}$, and (ii) comprehensive if for all $x, y \in \mathbb{R}_{+}^{n},[x>y$ and $x \in A] \Rightarrow y \in A$. For all $A \subseteq \mathbb{R}_{+}^{n}$, define the comprehensive hull of $A$, to be denoted by 
compA, as follows:

$$
\operatorname{comp} A \equiv\left\{z \in \mathbb{R}_{+}^{n} \mid z \leq x \text { for some } x \in A\right\} .
$$

As we noted in Introduction, a bargaining problem is a pair $(S, d)$ where $\varnothing \neq S \in \mathbb{R}^{n}$ and $d \in S$. Recall that $S$ is the bargaining set: the attainable set of utility allocations each of which can be achieved by the players through their joint action, and $d$ is interpreted as the threat or disagreement point: if the players fail to reach an agreement through their actions, the players will have to settle on $d$ for the bargaining problem $(S, d)$. Throughout our discussion, we make the following three assumptions: for each bargaining problem $(S, d)$ under consideration,

(i) $d$ is fixed,

(ii) $s \geq d$ for all $s \in S$, and $s \gg d$ for some $s \in S$,

(iii) each player's utility is cardinally measurable.

Since each player's utility function is cardinal so that the origin of each player's utility scale is arbitrary, we may without loss of generality normalize each player's utility function so that $\left(d_{1}, \cdots, d_{n}\right)=(0, \cdots, 0)$. As a consequence, we may normalize a bargaining problem $(S, d)$ to its 'normalized bargaining problem' $(S-\{d\}, 0)$, where $S-\{d\}=\{s-d \mid s \in S\}$. Given our assumptions above, each bargaining set $S-\{d\}$ is a non-trivial, compact and comprehensive $^{1}$ subsets of $\mathbb{R}_{+}^{n}$. Given this and with a slight abuse of notation, let $\Sigma$ be the set of all non-trivial, compact and comprehensive subsets of $\mathbb{R}_{+}^{n}$. Elements in $\Sigma$ are interpreted as (normalized) bargaining problems. Then, a bargaining solution $F$ on $\Sigma$ assigns a nonempty subset $F(A)$ of $A$ for every bargaining problem $A \in \Sigma$.

Let $\pi$ be a permutation of $N$. The set of all permutations of $N$ is denoted by $\Pi$. For all $x=\left(x_{i}\right)_{i \in N} \in \mathbb{R}_{+}^{n}$, let $\pi(x)=\left(x_{\pi(i)}\right)_{i \in N}$. For all $A \in \Sigma$ and any permutation $\pi \in \Pi$, let $\pi(A)=\{\pi(a) \mid a \in A\}$. For any $A \in \Sigma$, we say that $A$ is symmetric if $A=\pi(A)$ for all $\pi \in \Pi$.

For all $A \in \Sigma$ and all $i \in N$, let $m_{i}(A)=\max \left\{a_{i} \mid\left(a_{1}, \cdots, a_{i}, \cdots, a_{n}\right) \in A\right\}$. Therefore, $m(A) \equiv\left(m_{i}(A)\right)_{i \in N}$ is the ideal point of $\mathrm{A}$.

We now introduce the following classical solutions to bargaining problems in our context.

Definition 1: A bargaining solution $F$ over $\Sigma$ is

(1.1) the (symmetric) Nash solution, to be denoted as $F^{N}$, if for all $A \in \Sigma, F(A)=\{a \in$ $A \mid \prod_{i \in N} a_{i} \geq \prod_{i \in N} x_{i}$ for all $\left.x \in A\right\}$,

(1.2) the egalitarian solution, to be denoted as $F^{E}$, if for all $A \in \Sigma, F(A)=\left\{a \in A \mid a_{i}=a_{j}\right.$ for all $i, j \in N$ and there is no $x \in A$ such that $x \gg a$,

(1.3) the Kalai-Smorodinsky solution, to be denoted as $F^{K S}$, if for all $A \in \Sigma, F(A)=\{a \in$ $A \mid a_{i} / m_{i}(A)=a_{j} / m_{j}(A)$ for all $i, j \in N$ and there is no $x \in A$ such that $\left.x \gg a\right\}$,

\footnotetext{
${ }^{1}$ Given our above assumptions on bargaining problems, our notion of comprehensiveness of a bargaining set is equivalent to the notion of comprehensiveness of the bargaining set with respecc to the threat point $d$.
} 
(1.4) an asymmetric Nash solution, to be denoted as $F^{A N}$, if there exist $t_{1}>0, \cdots, t_{n}>0$ such that, for all $A \in \Sigma, F(A)=\left\{a \in A \mid \prod_{i \in N} a_{i}^{t_{i}} \geq \prod_{i \in N} x_{i}^{t_{i}}\right.$ for all $\left.x \in A\right\}$,

(1.5) a single-valued (symmetric) Nash solution, to be denoted as $F^{S V N}$, if $F$ is single-valued and for all $A \in \Sigma, F(A) \subseteq F^{N}(A)$,

(1.6) a single-valued (asymmetric) Nash solution, to be denoted as $F^{S V A N}$, if $F$ is singlevalued and for all $A \in \Sigma, F(A) \subseteq F^{A N}(A)$.

It may be helpful to relate the above definitions to the corresponding ones for a general bargaining problem $(S, d)$ : (1) an asymmetric Nash solution for $(S, d)$ is defined as $\left\{s^{*} \in S \mid\right.$ $\left.s^{*} \in \arg \max _{s \in S} \prod_{i=1}^{n}\left(s_{i}-d_{i}\right)^{t_{i}}\right\}$, (2) the egalitarian solution for $(S, d)$ is defined as the point $s^{e} \in S$ such that $s_{i}^{e}-d_{i}=s_{j}^{e}-d_{j}$ for all $i, j \in N$ and there is no $s \in S$ such that $s \gg s^{e}$, and (3) the Kalai-Smorodinsky solution for $(S, d)$ is defined as the point $s^{K S} \in S$ such that $\frac{s_{i}^{K S}-d_{i}}{m_{i}(S)-d_{i}}=\frac{s_{j}^{K S}-d_{j}}{m_{j}(S)-d_{j}}$ for all $i, j \in N$ and there is no $s \in S$ such that $s \gg s^{K S}$.

The (symmetric) Nash solution for nonconvex bargaining problems introduced above is identical to the one proposed by Kaneko (1980) and by Xu and Yoshihara (2006). It should be noted that, given that $\Sigma$ contains all non-trivial, compact and comprehensive bargaining problems, for any $A \in \Sigma$, the Nash solution, both symmetric and asymmetric, $F(A)$ can contain more than one alternative, while both the egalitarian and the Kalai-Smorodinsky solutions are singletons.

It may also be noted that, if the domain of bargaining problems, $\Sigma$, is enlarged to include bargaining problems each of which containing only a finite number of alternatives, then the egalitarian solution defined in Definition 1.2 as well as the Kalai-Smorodinsky solution defined in Definition 1.3 are no longer well-defined. For such larger domains, one would have to reformulate the solutions corresponding to the egalitarian and the KalaiSmorodinsky solutions. See Nagahisa and Tanaka (2002) for an alternative formulation of the Kalai-Smorodinsky solution for such a domain.

\section{$3 \quad$ Basic axioms}

As we noted in Introduction, the theoretical literature on bargaining problems is mainly axiomatic in that a solution satisfying a list of several reasonable axioms is shown to be unique so that this list of the axioms characterizes the solution. For this purpose, in this section, we present some of the axioms that are straightforward extensions of their counterparts in convex bargaining problems and that have been used for characterizing the classical solutions to nonconvex bargaining problems in the literature. We start with two efficiency type axioms that have been used in both convex and nonconvex bargaining problems (see, for example, Nash (1950), Kaneko (1980), Xu and Yoshihara (2006)).

Efficiency (E): For any $A \in \Sigma$ and any $a \in F(A)$, there is no $x \in A$ such that $x>a$.

Weak Efficiency (WE): For any $A \in \Sigma$ and any $a \in F(A)$, there is no $x \in A$ such that $x \gg a$. 
The next axiom is another familiar property often imposed on solutions to both convex and nonconvex bargaining problems (see, for example, Roth (1977a) and Zhou(1997)). It essentially requires that a solution should give players more utility than the threat or disagreement point. Formally, it can be stated as follows:

Strict Individual Rationality (SIR): For all $A \in \Sigma, x \in F(A) \Rightarrow x \gg \mathbf{0}$.

The next axiom is a variant of the familiar scale invariance property introduced in Nash (1950) for bargaining problems $(S, d)$ (see Roth (1977a) for the exact variant introduced for normalized bargaining problems).

Scale Invariance (SI): For all $A \in \Sigma$ and all $\alpha \in \mathbb{R}_{++}^{n}$, if $\alpha A=\left\{\left(\alpha_{i} a_{i}\right)_{i \in N} \mid a \in A\right\}$ then $F(\alpha A)=\left\{\left(\alpha_{i} a_{i}\right)_{i \in N} \mid a \in F(A)\right\}$.

Note that, since we study normalized bargaining problems (the disagreement point is $0 \in$ $\mathbb{R}_{+}^{n}$ ), appropriate positive linear transformations for a player can be confined to $\alpha_{i} u_{i}+\beta_{i}$ with $\beta_{i}=0$. See also Roth (1977a). (SI) is uncontroversial for convex bargaining problems. In the context of nonconvex bargaining problems, (SI) has its attractiveness as well whenever the underlying individuals' utilities are cardinally measurable, an implicit assumption for the standard bargaining problems. This is because, in many economic contexts of resource allocations, nonconvex problems may be derived from the nonconvexity of the underlying set of feasible allocations where the introduction of 'lottery' over feasible allocations is unavailable as in the deterministic framework of the classical resource allocation problems. In such contexts, (SI) has at least two interpretations; one is "utility-unit invariance," and the other is "independence of utility intensities" as discussed in Yoshihara (2003). Both interpretations are compatible with the nonconvexity of bargaining problems.

The next two axioms are natural generalizations of Nash's (1950) original symmetry axiom in the context of nonconvex bargaining problems.

Symmetry (S): For any $A \in \Sigma$, if $A$ is symmetric, then $[a \in F(A) \Rightarrow \pi(a) \in F(A)$ for all $\pi \in \Pi]$.

Strong Symmetry (SS): For any $A \in \Sigma$ and all $a \in A$, if $A$ is symmetric and $a \in F(A)$, then $a_{1}=\cdots=a_{n}$.

Symmetry is a natural generalization of Nash's original symmetry axiom to nonconvex problems. Strong Symmetry is a stronger requirement than Symmetry in that (SS) demands $a_{1}=\cdots=a_{n}$ whenever $A$ is symmetric and $a \in F(A)$, while (S) requires any permutation of $a$ be in $F(A)$ whenever $A$ is symmetric and $a \in F(A)$. It may be noted that, when restricted to convex bargaining problems, and bargaining solutions are required to be singlevalued mappings, the two symmetry axioms coincide with and are identical to Nash's original Symmetry axiom.

The last two axioms in this section are extensions of Nash's original Independence of Irrelevant Alternatives (IIA).

Contraction Independence (CI): For any $A, B \in \Sigma$, if $B \subseteq A$ and $B \cap F(A) \neq \varnothing$, then $F(B)=B \cap F(A)$. 
Weak Contraction Independence (WCI): For any $A, B \in \Sigma$, if $m(A)=m(B), B \subseteq A$ and $B \cap F(A) \neq \varnothing$, then $F(B)=B \cap F(A)$.

The axiom (CI) has been widely used in the literature of nonconvex bargaining problems (see, for example, Kaneko (1980), Xu and Yoshihara (2006)), and is the usual (IIA) for correspondence. (WCI) extends Roth's (1977b) axiom of "IIA other than ideal point" to correspondence, and is formally weaker than (CI): it restricts contractions to those problems that have the same ideal point. Various contraction type axioms used in bargaining theory are often motivated from rational choice perspectives (Chernoff (1954)). However, as discussed in $\mathrm{Xu}$ and Yoshihara (2008), they can also be interpreted on the basis of solidarity: when bargaining problems change from $A$ to $B$, the "utility gains" by any two players should not be in opposite directions-if one player gains from moving $A$ to $B$, then no player should become worse off from such a move; and if one player loses from moving $A$ to $B$, then no player should gain from such a move.

\section{Rationalizability of bargaining solutions}

Bargaining solutions can be interpreted differently. A solution can be viewed as representing the majority preferences of the committee whose charge is to come up with a fair arbitration scheme (see Mariotti, 1999, Xu and Yoshihara, 2013). Secondly, a solution to bargaining problems may be thought to reveal the preferences of the players involved as a group, and thus, the behavior of a solution may be linked to 'revealed group preference' (Peters and Wakker, 1991, Xu and Yoshihara, 2013). Finally, a bargaining solution can be viewed as choices or recommendations made by a rational benevolent designer or social planner who "is able to correctly observe the player' utility levels, who is without own interest" (Trockel (1999)) on behalf of the players. In these interpretations, it is sensible to ask and examine the question whether a solution can be rationalized by a well-behaved preference relation. In the theory of rational choice concerning a single individual, there is a huge literature studying rationalizability of an individual's choices. In the literature on bargaining problems, the rationalizability of solutions to convex bargaining problems has been fruitfully studied by several authors including Peters and Wakker (1991), Bossert (1994), Sänchez (2000), and Trockel (1999). As for research on the rationalizability of solutions to nonconvex bargaining problems, there are recent contributions by Denicolò and Mariotti (2000), and Xu and Yoshihara (2013). In what follows and before we study classical solutions to nonconvex bargaining problems, we study rationalizability of solutions to nonconvex bargaining problems.

The rationalizability of a solution is formally defined as follows:

Definition 2: A bargaining solution $F$ over $\Sigma$ is rationalizable if there exists a reflexive, complete and transitive binary relation $R$ over $\mathbb{R}_{+}^{n}$ such that, for all $A \in \Sigma, F(A)=\{x \in$ $A \mid x R y$ for all $y \in A\}$.

In rational choice theory, various consistency in terms of expansion and contraction axioms have been introduced and used for discussions of rationalizability of individual choices. (CI) introduced in Section 3 is one of such axioms. In what follows, we introduce two fairly weak consistency axioms for our purpose (Xu and Yoshihara (2013)). 
Binary Contraction Independence (BCI): For all $A \in \Sigma$ and all $x, y \in A$, if $\{x, y\} \cap$ $F(A) \neq \varnothing$ then $F(A) \cap\{x, y\} \subseteq F(\operatorname{comp}\{x, y\})$.

Binary Expansion Independence (BEI): For all $A \in \Sigma$ and all $x, y \in A$, if $\{x, y\}=$ $F(\operatorname{comp}\{x, y\})$, then $[x \in F(A) \Leftrightarrow y \in F(A)]$.

The idea behind a contraction consistency axiom in the context of rationalizability of bargaining solutions is the following: given two bargaining problems, $A$ and $B$, in which $A$ is a subset of $B$, and suppose that a point $x$ chosen from $B$ as a solution to $B$ continues to be available in $A$, then $x$ should continue to be a solution to $A$ provided certain restrictions are satisfied. (BCI) stipulates that, for any two points $x$ and $y$ in a bargaining problem $A$, if either $x$ or $y$ is part of the solution to $A$, then the common points in $\{x, y\}$ and the solution to $A$ must be contained in the solution to the problem given by the comprehensive hull of $x$ and $y$. (BCI) is clearly weaker than (CI) and is introduced in Xu and Yoshihara (2013), though its origin goes back to Herzberger (1973) and Sen (1971).

Similarly, the idea behind an expansion consistency axiom in the present context can be explained as follows: given two bargaining problems, $A$ and $B$, in which $A$ is a subset of $B$, and suppose that two points $x$ and $y$ chosen from $A$ as solutions to $A$ continue to be available in $B$, then, if one is a solution to $B$ then the other must be a solution to $B$ as well (and if one is no longer a solution to $B$ then the other cannot be a solution to $B$ either). (BEI) requires that, whenever the solution to the problem $\operatorname{comp}\{x, y\}$ consists of both $x$ and $y$, then, for any problem $A$ containing both $x$ and $y$, either $[x$ and $y$ are both chosen as solutions to $A$ ] or [neither $x$ nor $y$ is chosen as a solution to $A$ ]. (BEI) is formally weaker than the corresponding property $\beta$ in the rational choice literature (see Sen, 1971).

With the help of the above two consistency axioms, we have the following result which is due to $\mathrm{Xu}$ and Yoshihara (2013).

Theorem 1. Let a solution $F$ over $\Sigma$ satisfy (E) and (SIR). Then, $F$ satisfies (BCI) and (BEI) if and only if $F$ is rationalizable.

Sketch of the proof. Rather than giving a formal proof of Theorem 1, we present a sketch of the proof here. For a formal proof, see Xu and Yoshihara (2013). Let a solution $F$ over $\Sigma$ satisfy (E), (SIR), (BCI) and (BEI). Define a binary relation $R$ over $\mathbb{R}_{+}^{n}$ as follows: for all $x, y \in \mathbb{R}_{+}^{n}$,

if $x=y$, then $x R x$; if $x \neq y$, then $x R y \Leftrightarrow[x \in F(\operatorname{comp}\{x, y\})]$ or

$[y \notin F(A)$ for all $A \in \Sigma$ with $x, y \in A]$.

It can be checked that the binary relation $R$ defined above is well-defined, and is reflexive, transitive and complete. To show that $R$ rationalizes $F$, we need to check if the following statement is true:

for all $A \in \Sigma: F(A)=\{a \in A \mid a R x$ for all $x \in A\}$

Take $A \in \Sigma$ and let $a \in F(A)$. (SIR) implies that, for any $x \in A$ with $x_{i}=0$ for some $i \in N$, we have $x \notin F(A)$ and therefore $a R x$ in this case. For any $x \in A$ with $x \gg \mathbf{0}$, noting that $a \in F(A)$, by (BCI), it follows that $a \in F(\operatorname{comp}\{a, x\})$ implying that $a R x$. Therefore, $F(A) \subseteq\{a \in A \mid a R x$ for all $x \in A\}$. 
On the other hand, if, for some $a \in A$ such that $a R x$ for all $x \in A$ but $a \notin F(A)$, then, noting that $F(A) \neq \varnothing$, there must be some $z \in F(A)$. By (BCI), $z \in F(\operatorname{comp}\{a, z\})$. Since $a R z$, equivalently $a \in F(\operatorname{comp}\{a, z\})$, we then have $\{a, z\}=F(\operatorname{comp}\{a, z\})$. By (BEI), it follows that $a \in F(A)$, a contradiction. Therefore, for all $a \in A$ with $a R x$ for all $x \in A$, we must have $a \in F(A)$; that is, $\{a \in A \mid a R x$ for all $x \in A\} \subseteq F(A)$.

Therefore, $F(A)=\{a \in A \mid a R x$ for all $x \in A\}$. $\diamond$

Remark 1. It may be noted that when a solution $F$ is restricted to be single-valued, (BEI) is vacuous, and consequently, the following result holds:

Theorem 2. Let a solution $F$ over $\Sigma$ be single-valued and satisfy (E) and (SIR). Then, $F$ satisfies (BCI) if and only if $F$ is rationalizable.

Denicolò and Mariotti (2000) obtain a similar result to Theorem 2 by using (CI) and with a larger domain of bargaining problems, some of which may contain finitely many points.

\section{Nash solution: refinement and extension}

In this section, we discuss various axiomatic characterizations of the Nash solution and its refinement and extension in the context of nonconvex bargaining problems. For ease of presentation, we divide this section into three subsections: we first present results on the Nash solution, then the results relating to its refinements and extensions, and finally we discuss results of single-valued solution.

\subsection{Nash solution}

We begin with a characterization of the (symmetric) Nash solution obtained in $\mathrm{Xu}$ and Yoshihara (2006).

Theorem 3: A bargaining solution $F$ over $\Sigma$ is the Nash solution if and only if it satisfies (E), (S), (SI) and (CI).

Sketch of the proof. We illustrate the main idea of the proof by focusing on $n=2$. For a formal proof, see $\mathrm{Xu}$ and Yoshihara (2006). A crucial step in establishing the result is to show that,

Step 1: for all $x, y \in \mathbb{R}_{+}^{2}, x_{1} x_{2}=y_{1} y_{2}>0 \Rightarrow F(\operatorname{comp}\{x, y\})=\{x, y\}$

Take $x, y \in \mathbb{R}_{+}^{2}$ with $x_{1} x_{2}=y_{1} y_{2}>0$. Define $\alpha \in \mathbb{R}_{+}^{2}$ by: $\alpha_{1}=1, \alpha_{2}=\frac{x_{1}}{y_{2}}$. Then, $\alpha\{x\}=$ $\left(x_{1}, x_{2} \frac{x_{1}}{y_{2}}\right)=\left(x_{1}, \frac{y_{1} y_{2}}{y_{2}}\right)=\left(x_{1}, y_{1}\right)$ and $\alpha\{y\}=\left(y_{1}, y_{2} \frac{x_{1}}{y_{2}}\right)=\left(y_{1}, x_{1}\right)$. Note that $\alpha\{x\}$ is a permutation of $\alpha\{y\}$. Consider the bargaining problem $A=\operatorname{comp}\left\{\left(\alpha_{1} x_{1}, \alpha_{2} x_{2}\right),\left(\alpha_{1} y_{1}, \alpha_{2} y_{2}\right)\right\}=$ $\operatorname{comp}\left\{\left(x_{1}, y_{1}\right),\left(y_{1}, x_{1}\right)\right\}$. Note that $\left(x_{1}, y_{1}\right)$ and $\left(y_{1}, x_{1}\right)$ are the only two efficient point in $A$ and that $A$ is symmetric. By (E) and (S), we then must have $F(A)=\left\{\left(x_{1}, y_{1}\right),\left(y_{1}, x_{1}\right)\right\}$. By (SI), it then follows that $\{x, y\}=F(\operatorname{comp}\{x, y\})$.

With the help of Step 1, we can show the following step: 
Step 2: for all $x, z \in \mathbb{R}_{+}^{2}, x_{1} x_{2}>z_{1} z_{2} \Rightarrow\{x\}=F(\operatorname{comp}\{x, z\})$

Take $x, z \in \mathbb{R}_{+}^{2}$ with $x_{1} x_{2}>z_{1} z_{2}$. Note that there exists $\left(t_{1}, t_{2}\right)$ with $t_{1}>0, t_{2}>0$ such that $\left(z_{1}+t_{1}\right)\left(z_{2}+t_{2}\right)=x_{1} x_{2}$. From Step 1, $\left\{x,\left(z_{1}+t_{1}, z_{2}+t_{2}\right)\right\}=F\left(\operatorname{comp}\left\{x,\left(z_{1}+t_{1}, z_{2}+t_{2}\right)\right\}\right)$. On the other hand, it must be true that $\operatorname{comp}\{x, z\} \subseteq\left(\operatorname{comp}\left\{x,\left(z_{1}+t_{1}, z_{2}+t_{2}\right)\right\}\right.$. By (CI), it then follows that $\{x\}=F(\operatorname{comp}\{x, z\})$.

Consider $A \in \Sigma$. Since $A$ is compact, there must be $a \in A$ with $a_{1} a_{2} \geq x_{1} x_{2}$ for all $x \in A$. Consider $b \in A$ with $b_{1} b_{2}<a_{1} a_{2}$. If $b \in F(A)$, then by $(\mathrm{CI}), b \in F(\operatorname{comp}\{a, b\})$, which would contradict Step 2. Using a similar argument, it must be the case that, for any $x \in A$, if $x_{1} x_{2}=a_{1} a_{2}$, then $x \in F(A)$. Consequently, $F(A)=\left\{x \in \mathbb{R}_{+}^{2} \mid x_{1} x_{2} \geq z_{1} z_{2}\right.$ for all $z \in A\} . \diamond$

The axioms used in characterizing the Nash solution in Theorem 3 are straightforward extensions of the original axioms used by Nash (1950) for convex problems to nonconvex problems. Maintaining (E), (SI) and (CI), Zambrano (2016) extends the original symmetry axiom to the following axiom and uses it together with (E), (SI) and (CI) to characterize the Nash solution. Before introducing the modified symmetry, a definition is needed.

Let $A \in \Sigma$ and let $x, y \in A$ with $x \neq y$. We say that $y$ is comparably more symmetric than $x$ if $y=\lambda x+(1-\lambda) \pi(x)$ for $\lambda \in(0,1)$ and $\pi$ is a permutation of $N$.

Preference for Symmetry (Sy): For all symmetric $A \in \Sigma$, if $x, y \in A$ are such that $y$ is comparably more symmetric than $x$, then $x \notin F(A)$.

Note that if $y$ is comparably more symmetric than $x$, then $y$ lies on the line segment linking $x$ and a permutation of $x$. This observation suggests the following: If a solution to a bargaining problem $A$ is viewed as consisting of those utility allocations each of which maximizes a 'selection function' of utility allocations over $A$, then the axiom (Sy) requires a certain degree of quasiconcavity on the selection function and that the selection function exhibits inequality aversion.

The following result is due to Zambrano $(2016)^{2}$ :

Theorem 4. A bargaining solution $F$ over $\Sigma$ is the Nash solution if and only if it satisfies (E), (Sy), (SI) and (CI).

It may be noted that, in view of our remark concerning the property of inequality aversion exhibited by (Sy), there is a certain amount of inequality aversion embedded in the Nash solution.

Kaneko (1980) uses the following continuity property to characterize the symmetric Nash solution.

Continuity (CON): Let $\left\{A^{K}\right\}$ be a sequence of sets in $\Sigma$ which converges to $A^{0} \in \Sigma$ (in the sense of the Hausdorf metric for subsets). Let $\left\{a^{k}\right\}$ be any sequence such that $\lim _{k \rightarrow \infty} a^{k}=a^{0}$ and $a^{k} \in F\left(A^{k}\right)$ for all $k=1,2, \cdots$, . Then $a^{0} \in F\left(A^{0}\right)$.

\footnotetext{
${ }^{2}$ To be exact, Zambrano (2016) considers a larger domain of bargaining problems than $\Sigma$-his domain may contain bargaining problems with a finitely many points.
} 
The following characterization result is due to Kaneko (1980).

Theorem 5. A solution $F$ over $\Sigma$ satisfies (E), (SI), (S), (CI) and (CON) if and only if $F=F^{N}$.

It may be of interest to note that the axiom $(\mathrm{CON})$ in Theorem 5 is redundant as shown in Theorem 2 that the (symmetric) Nash solution is characterized by (E), (SI), (S) and (CI).

In a related contribution, $\mathrm{Xu}$ and Yoshihara (2013) introduce the following continuity property of a bargaining solution and characterize an asymmetric Nash solution (Theorem 6 below).

Weak Continuity (WCON): For any $x, y \in \mathbb{R}_{+}^{n}$ with $x \neq y$, if $\{x\}=F(\operatorname{comp}\{x, y\})$, then there exists $\epsilon>0$ such that, for all $z \gg 0$ and all $z^{\prime} \in \mathbb{R}_{+}^{n}$,

$$
\|z-x\|<\epsilon \Rightarrow[\{z\}=F(\operatorname{comp}\{y, z\})]
$$

and

$$
\left\|z^{\prime}-y\right\|<\epsilon \Rightarrow\{x\}=F\left(\operatorname{comp}\left\{x, z^{\prime}\right\}\right)
$$

Note that (WCON) is weaker than (CON). With the help of (WCON), Xu and Yoshihara (2013) obtain the following result:

Theorem 6. A solution $F$ over $\Sigma$ satisfies (E), (SIR), (BCI), (BEI), (SI) and (WCON) if and only if it is an asymmetric Nash solution.

Outline of the proof. Let a solution $F$ over $\Sigma$ satisfy (E), (SIR), (BEI), (SI) and (WCON). From Theorem $1, F$ is rationalizable by a reflexive, transitive and complete binary relation $R$ over $\mathbb{R}_{+}^{n}$. (WCON) implies that $R$ is continuous. Then, from (SI), $R$ satisfies the following property: for all $x, y \in \mathbb{R}_{++}^{n}$ and all $\lambda \in \mathbb{R}_{++}^{n}, x R y \Leftrightarrow\left(\lambda_{1} x_{1}, \cdots, \lambda_{n} x_{n}\right) R\left(\lambda_{1} y_{1}, \cdots, \lambda_{n} y_{n}\right)$. Following Tsui and Weymark (1997) and Xu (2002), there exist $t_{1}, \cdots, t_{n}$ such that, for all $x, y \in \mathbb{R}_{++}^{n}, x R y \Leftrightarrow \prod_{i=1}^{n} x_{i}^{t_{i}} \geq \prod_{i=1}^{n} y_{i}^{t_{i}}$. (E) implies that $t_{1}>0, \cdots, t_{n}>0$. Note that if $y_{i}=0$ for some $i \in N$, then $x R y$. Therefore, $R$ can be represented by a Cobb-Douglas function, and $F$ is thus an asymmetric Nash solution. $\diamond$

The (symmetric) Nash solution can be characterized by requiring (S) for a solution. Indeed, as reported in Xu and Yoshihara (2013), the following result holds.

Theorem 7. A solution $F$ over $\Sigma$ satisfies (WE), (SI), (S), (BCI), and (BEI) if and only if $F$ is the Nash solution.

To conclude the discussion of this subsection, it may be noted that, Herrero (1989) considers solutions to two-person 'smooth' bargaining problems and characterizes the Nash solution by (SIR), (E), (SI), (CI), Nash's original symmetry axiom (applied to symmetric convex bargaining problems only), and a lower semi-continuity property of a solution. Moreover, Mariotti (2000) studies an alternative characterization of the Nash solution in the domain 
$\Sigma$. He introduces an axiom called Maximal Symmetry requiring that whenever a solution selects a point, say $x$, from a bargaining problem, it must also select any permutation of $x$ if the permutation is in the bargaining problem. In two other papers, Mariotti (1998, 1999) studies characterizations of the Nash solution with a larger domain that also includes finite bargaining problems. Mariotti (1999) introduces a new axiom called Suppes-Sen Proofness requiring that if a bargaining problem contains a point $x$ and a permutation of $x$ such that either $x$ or the permutation of $x$ is weakly dominated by another point in the problem, then the solution should never pick either $x$ or its permutation as solutions, and axiomatically characterizes the Nash solution by this new axiom together with (SI).

\subsection{Refinement and extension}

From the results of the last subsection, it is clear that, in general, the solution $F(A)$ to a bargaining problem $A$ is multi-valued $-F(A)$ may contain more than one element due to the possibility that $A$ can be nonconvex. In this subsection, we discuss possible refinements and extensions of the Nash solution proposed in the literature. We start with a procedural method of eliminating certain elements from the original Nash solution set proposed in Xu and Yoshihara (2019).

First, we introduce two definitions. A problem $A \in \Sigma$ is called simple if there are $x, y \in A$ such that $A=\operatorname{comp}\{x, y\}$. For all $x \in \mathbb{R}_{+}^{n}$, let $\bar{x}=\max \left\{x_{i} \in \mathbb{R}_{+}^{n} \mid i=1, \cdots, n\right\}$ and $\underline{x}=\min \left\{x_{i} \in \mathbb{R}_{+}^{n} \mid i=1, \cdots, n\right\}$.

Definition 3: A bargaining solution over $\Sigma$ is the equitable Nash solution, to be denoted by $F^{E N}$, if for all $A \in \Sigma, F^{E N}(A)=\left\{a \in F^{N}(A) \mid \min _{i \in N}\left\{\frac{a_{i}}{m_{i}(A)}\right\} \geq \min _{i \in N}\left\{\frac{x_{i}}{m_{i}(A)}\right\}\right.$ for all $\left.x \in F^{N}(A)\right\}$.

According to the solution $F^{E N}$, for any given problem $A$, we first find out the Nash solution, $F^{N}(A)$, to the bargaining problem $A$, and then, from the Nash solution set $F^{N}(A)$, we select all the points lying on the highest indifference surface given by $\min \left\{a_{1} / m_{1}(A), \cdots, a_{n} / m_{n}(A)\right\}$. Viewed differently, in the process of finding out the solution to a bargaining problem $A$, it applies two 'maximizers', $\max _{x \in A} x_{1} \cdots x_{n}$, and $\max _{x \in A} \min \left\{x_{1} / \min _{1}(A), \cdots, x_{n} / \min _{n}(A)\right\}$, sequentially. $F^{E N}$ can thus be viewed as a procedural refinement of the Nash solution.

To see that $F^{E N}$ refines the Nash solution to nonconvex bargaining problems, consider the bargaining problem $A=\operatorname{comp}\{(3,12),(6,6),(9,4)\}$. Note that the Nash solution to $A$ is given by $\{(3,12),(6,6),(9,4)\}$. Note that $m_{1}(A)=9$ and $m_{2}(A)=12$. Then, $\left(\frac{3}{9}, \frac{12}{12}\right)=\left(\frac{1}{3}, 1\right)$, $\left(\frac{6}{9}, \frac{6}{12}\right)=\left(\frac{2}{3}, \frac{1}{2}\right)$, and $\left(\frac{9}{9}, \frac{4}{12}\right)=\left(1, \frac{1}{3}\right)$. Note that $\min \left\{\frac{2}{3}, \frac{1}{2}\right\}=\frac{1}{2}>\min \left\{\frac{1}{3}, 1\right\}$. Therefore, $F^{E N}(A)=\{(6,6)\}$. The intuitive idea here is that, the three outcomes lie on the same 'indifference curve' given by $x_{1} x_{2}=36$, and, for both players, the outcome $(6,6)$ lies 'inbetween' $(3,12)$ and $(9,4)$, suggesting that the outcome $(6,6)$ is 'more equitable' than the two 'more extreme' outcomes $(3,12)$ and $(9,4)$.

To characterize $F^{E N}$, we consider the following two axioms that are weaker versions of the Weak Axiom of Revealed Preference (WARP) proposed in the literature on rational choice (Samuelson, 1938, 1947). 
Binary Weak Axiom of Revealed Preference 1 (BWARP1): For all $x, y, z \in \mathbb{R}_{+}^{n}$ with $y>z$, if $\{x, y\}=F(\operatorname{comp}\{x, y\})$, then $z \notin F(A)$ for any $A \in \Sigma$ with $x, z \in A$.

Binary Weak Axiom of Revealed Preference 2 (BWARP2): For all $x, z \in \mathbb{R}_{+}^{n}$, if $\{x\}=F(\operatorname{comp}\{x, z\})$, then $z \notin F(A)$ for any $A \in \Sigma$ with $x, z \in A$.

The two axioms above are simple as they each involve the information about the solution to simple bargaining problems and are proposed in Xu and Yoshihara (2019). In particular, (BWARP1) requires that, if an alternative $z$ is "revealed to be worse" than another alternative $x$ through a "pairwise comparison" involving $x$ and $y$ where $y$ vectorially dominates $z$, then $z$ should not be chosen as a solution as long as $x$ is available. (BWARP2) requires that, if an alternative $z$ is "revealed to be worse" than another alternative $x$ directly, then $z$ should not be chosen as a solution as long as $x$ is available. Both are weaker versions of (WARP).

The next axiom reflects an equity concern in making a solution recommendation for bargaining problems and essentially requires that, if two points are "informationally equivalent", that is, if both are chosen part of the solutions to the simple bargaining problem formed by the comprehensive hull of them, but one is 'more equitable' than the other in the spirit of the Hammond equity principle (Hammond, 1976) in a symmetric bargaining problem, then the less equitable point should not be a part of the solution of this symmetric problem. Formally, it may be stated as follows:

Equity Principle (EP): For all non-simple and symmetric $A \in \Sigma$ and for all $x \in A$, if there exists $y \in A$ such that $\{x, y\}=F(\operatorname{comp}\{x, y\})$ and $(\underline{x}, \cdots, \underline{x})<y<(\bar{x}, \cdots, \bar{x})$, then $x \notin F(A)$.

It may be noted that the axiom (EP) appeals to 'inequality aversion' regarding how a solution selects solution candidates for a non-simple and symmetric bargaining problem. As we remarked earlier, the axiom (Sy) can be viewed as exhibiting an inequality aversion also. However, the ways in which the two axioms, (EP) and (Sy), are applied for this purpose are very different: (EP) is applied to two points that are deemed as 'informationally equivalent' (in a pairwise comparison, they both are solution candidates), while (Sy) is applied to two points in which one is comparably more symmetric than the other. Consequently, the bite of (EP) is more about how to refine a solution and the gist of (Sy) is more about how to select a solution candidate to begin with.

With the help of the three new axioms, we can now state the following result ( $\mathrm{Xu}$ and Yoshihara, 2019).

Theorem 8. A solution F satisfies (E), (S), (SI), (BWARP1), (BWARP2), (WCI), and (EP) if and only if $F=F^{E N}$.

Intuitive idea behind the proof. Instead of giving a proof of this theorem, we illustrate, using our earlier example, why the solution to the problem $\operatorname{comp}\{(3,12),(6,6),(9,4)\}$ is given by $\{(6,6)\}$. For a formal proof of Theorem 8, see Xu and Yoshihara (2019).

We first use (SI) to transform the problem $A=\operatorname{comp}\{(3,12),(6,6),(9,4)\}$ to $\alpha A=$ $\operatorname{comp}\{(12,36),(24,18),(36,12)\}$, where $\alpha=(4,3)$. Consider the problem 
$B=\operatorname{comp}\{(12,36),(24,18),(18,24),(36,12)\}$. Note that $B$ is symmetric and non-simple. Following a similar intuition behind the characterization of the Nash solution, it can be checked that, for any $x, y \in\{(12,36),(24,18),(18,24),(36,12)\}, F(\operatorname{comp}\{x, y\})=\{x, y\}$. Note that $(24,18)$ and $(18,24)$ are such that $12<18<24<36,12=\min \{12,36\}$ and $36=$ $\max \{12,36\}$. Therefore, by $(\mathrm{EP}),(12,36) \notin F(B)$, and $(36,12) \notin F(B)$. Then, by $(\mathrm{E})$, either $(24,18) \in F(B)$ or $(18,24) \in F(B)$. By $(\mathrm{S}), F(B)=\{(24,18),(18,24)\}$. (WCI) then implies that $\{(24,18)\}=F(\{(12,36),(24,18),(36,12)\}) .\{(6,6)\}=F(\operatorname{comp}\{(3,12),(6,6),(9,4)\})$ then follows from $(\mathrm{SI})$. $\diamond$

The above argument on the bargaining problem $A=\operatorname{comp}\{(3,12),(6,6),(9,4)\}$ also suggests the possibility of an anonymous and inequitable bargaining solution $F^{\prime}$ which satisfies all of the axioms in Theorem 8 except (EP), such that $F^{\prime}(\operatorname{comp}\{(3,12),(6,6),(9,4)\})=$ $\{(3,12),(9,4)\}$.

In a contribution by Peters and Vermeulen (2012), they show that any bargaining solution $F$ satisfying (WE), (SI) and (CI) must be a refinement of certain asymmetric Nash bargaining solution.

Theorem 9. A solution $F$ satisfies (WE), (SI) and (CI) if and only if, for some $\alpha \in \mathbb{R}_{+}^{n}, \alpha \neq$ 0:

$$
\text { for all } A \in \Sigma, F(A) \subseteq\left\{a \in A \mid \prod_{i \in N} a_{i}^{\alpha_{i}} \geq \prod_{i \in N} x_{i}^{\alpha_{i}}, x \in A\right\} \text {. }
$$

Therefore, (WE), (SI) and (CI) together give us a set of basic axioms for a particular way of refining Nash solution for nonconvex bargaining problems. Note that $F^{E N}$ does not satisfy (CI) in general suggesting that the refining method embedded in $F^{E N}$ is different from the one embedded in a solution $F$ satisfying (WE), (SI) and (CI).

Points selected by a solution $F$ satisfying (WE), (SI) and (CI) can be further partitioned into three different groups, $L, D$, and $R$, by considering $F(\operatorname{comp}\{x, e\})$ where $e=(1, \cdots, 1)$ (Peters and Vermeulen, 2012): the $L$ group consists of those $x$ such that $\{x\}=F(\operatorname{comp}\{x, e\})$, the $D$ group consists of those $x$ such that $\{x, e\}=F(\operatorname{comp}\{x, e\})$, and the $R$ group consists of those $x$ such that $\{e\}=F(\operatorname{comp}\{x, e\})$. Further refinement of a solution satisfying (WE), (SI) and (CI) can be done by applying specific groupings of $L$, $D$, or $R$. See Peters and Vermeulen (2012) for further results along these lines.

To conclude this subsection, we discuss an extension of the Nash solution proposed by Conley and Wilkie (1996). We first introduce a notation. For a set $A \subseteq \mathbb{R}_{+}^{n}$, the convex hull of $A$, to be denoted by $\operatorname{con}(A)$, is defined as the smallest convex set containing $A$.

Definition 4: A bargaining solution $F$ over $\Sigma$ is an extension of the Nash solution, to be denoted by $F^{N *}$, if, for any $A \in \Sigma, F(A)$ consists of unique weakly efficient point that is in $A$ and that lies on the line segment linking the disagreement point $\mathbf{0}$ and the unique point in $F^{N}\left(\operatorname{comp}\left(\operatorname{con}\left(F^{N}(A)\right) \cup\{\mathbf{0}\}\right)\right)$.

For their characterization, Conley and Wilkie (1996) introduce the following monotonicity type axiom: 
Ethical Monotonicity (EM): For all $A, A^{\prime} \in \Sigma$ with $\{a\}=F(A)$ and $\left\{a^{\prime}\right\}=F\left(A^{\prime}\right)$, if $A^{\prime} \subseteq A$ and $a \in \operatorname{con}\left(A^{\prime}\right)$, then $a \geq a^{\prime}$.

They then obtain the following result (Conley and Wilkie, 1996):

Theorem 10. Suppose $F$ is single-valued. Then, $F$ over $\Sigma$ satisfies (WE), (S), (SI), (EM), $(\mathrm{CON})$ if and only if $F=F^{N *}$.

Instead of giving a proof, we illustrate how the solution can be obtained and how this solution is different from Nash solution. We first note that, if $\operatorname{con}\left(F^{N}(A)\right)$ is convex, $\operatorname{comp}\left(\operatorname{con}\left(F^{N}(A)\right) \cup\{\mathbf{0}\}\right)$ is convex as well. Consequently, $F^{N}\left(\operatorname{comp}\left(\operatorname{con}\left(F^{N}(A)\right) \cup\{\mathbf{0}\}\right)\right)$ is single-valued. Therefore, $F^{N *}$ is single-valued. Note also that, if $A$ is convex, then $F^{N}(A)=F^{N *}$. When $A$ is nonconvex, it is possible that $F^{N *}(A)$ is not a subset of $F^{N}(A)$. Consider the following example. Let $A=\operatorname{comp}(\{(1,36),(36,1),(2,18)\})$. Then, $F^{N}(A)=$ $\{(1,36),(36,1),(2,18)\}$, and $\operatorname{comp}\left(\operatorname{con}\left(F^{N}(A)\right) \cup\{(0,0)\}\right)=\left\{x \in \mathbb{R}_{+}^{2} \mid x_{1}+x_{2} \leq 37\right.$, $\left.x_{1} \leq 36, x_{2} \leq 36\right\}$. Consequently, $F^{N}\left(\operatorname{comp}\left(\operatorname{con}\left(F^{N}(A)\right) \cup\{(0,0)\}\right)\right)=\{(18.5,18.5)\}$, and $F^{N *}(A)=\{(2,2)\}$.

\subsection{Single-valued Nash solution}

In this subsection, we briefly discuss the issue of single-valued symmetric and asymmetric Nash solution. We start by discussing a result obtained in Zhou (1997). He uses the following version of (SI) to obtain the result:

Restricted Scale Invariance (RSI): For all $A \in \Sigma$ with $A$ being convex and all $\alpha \in \mathbb{R}_{++}^{n}$, if $\alpha A=\left\{\left(\alpha_{i} a_{i}\right)_{i \in N} \mid a \in A\right\}$ then $F(\alpha A)=\left\{\left(\alpha_{i} a_{i}\right)_{i \in N}: a \in F(A)\right\}$.

Note that in (RSI), 'scale invariance' is applicable to convex bargaining problems only. This is faithful to Nash's (1951) original idea about individual utility functions. With (RSI) in hand, Zhou (1997) obtains the following result.

Theorem 11. Suppose $F$ over $\Sigma$ is single-valued. Then, if $F$ satisfies (SIR), (RSI) and (CI), then $F=F^{S V A N}$.

Denicolò and Mariotti (2000), using a slightly different set of axioms, obtain the following result:

Theorem 12. Suppose $F$ over $\Sigma$ is single-valued. Then, if $F$ satisfies (E), (SI) and (CI), then $F=F^{S V A N}$.

For two-player 'star-shaped' bargaining problems'3 , Qin, Tan and Wong (2017) give an axiomatic characterization of a particular single-valued symmetric Nash solution, the 'dictatorial selection' of the Nash solution. For their result, they introduce the following property, which is weaker than Nash's (1951) original symmetry axioms.

Weak Symmetry (WS): For $A=\left\{x \in \mathbb{R}_{+}^{2} \mid x_{1}+x_{2} \leq 1\right\}, a \in F(A) \Rightarrow a_{1}=a_{2}$.

\footnotetext{
${ }^{3} \mathrm{~A}$ bargaining problem $A$ is star-shaped if, for all $x \in A$ and all $t \in[0,1], t x \in A$.
} 
With (WS), Qin, Tan and Wong (2017) obtain the following result ${ }^{4}$ :

Theorem 13. Suppose $n=2$ and a solution $F$ over $\Sigma$ is single-valued. Then, $F$ satisfies (SIR), (SI), (CI) and (WS) if and only if, for some $i \in\{1,2\}$, for all $A \in \Sigma$,

$$
F(A)=\arg \max _{a \in F^{N}(A)} a_{i}
$$

Therefore, for single-valued solutions to nonconvex bargaining problems with two players, there are exactly two each being a dictatorial selection of Nash product maximizers. To use our earlier example in which $A=\operatorname{comp}\{(3,12),(6,6),(9,4)\}$, we have either $F(A)=\{(9,4)\}$ or $F(A)=\{(3,12)\}$. And further, if $F(A)=\{(9.4)\}$, then player 1 "dictates" the selection of the solution point in $F^{N}(S)$ for any bargaining problem $S \in \Sigma$. Likewise, if $F(A)=\{(3,12)\}$, then the selection of the solution point in $F^{N}(S)$ for any bargaining problem $S \in \Sigma$ is exclusively based on player 2's utility.

\section{Kalai-Smorodinsky solution}

The Kalai-Smorodinsky solution to nonconvex bargaining problems is single-valued. We present a simple and straightforward characterization result due to Xu and Yoshihara (2006).

Theorem 14: A bargaining solution $F$ over $\Sigma$ is the Kalai-Smorodinsky solution if and only if it satisfies (WE), (SS), (SI) and (WCI).

Sketch of the proof. We give an outline of the proof for $n=2$. See $\mathrm{Xu}$ and Yoshihara (2006) for a formal proof.

Consider $A \in \Sigma$. By (SI), we assume that $m_{1}(A)=m_{2}(A)=m>0$. Let $a \in A$ be such that $a_{1}=a_{2}$ and weakly efficient in $A$. Consider the bargaining problem $B=$ $\operatorname{comp}\left\{\left(a_{1}, m\right),\left(m, a_{2}\right)\right\}$. Note that $B$ is symmetric, $A \subseteq B$, and $\left(a_{1}, a_{2}\right) \in B$ is weakly efficient in $B$. By (SS) and (WE), $\{a\}=F(B)$. By (WCI), $\{a\}=F(A)$.

It may be noted that, in a smaller domain that contains certain nonconvex bargaining problems, Anant, Mukherji and Basu (1990) characterizes the Kalai-Smorodinsky solution for two players by (E), (SS), (SI), and a monotonicity type axiom.

Conley and Wilkie (1991) consider a similar domain to $\Sigma$, and present an alternative characterization of the Kalai-Smorodinsky solution. Instead of using a variant of (CI), they adopt the monotonicity axiom used by Kalai and Smorodinsky (1975) in the context of convex bargaining problems.

Weak Monotonicity (WMON): For all $A, B \in \Sigma$ with $A \subseteq B$, if $m(A)=m(B)$, then $F(B) \geq F(A)$.

The following result is due to Conley and Wilkie (1991):

\footnotetext{
${ }^{4}$ The result can be easily extended from the domain of star-shaped bargaining problems to $\Sigma$.
} 
Theorem 15: A bargaining solution $F$ over $\Sigma$ is the Kalai-Smorodinsky solution if and only if it satisfies (WE), (SS), (SI) and (WMON).

Sketch of the proof. Consider $n=2$. Given (SI), consider $A \in \Sigma$ with $m(A)=(1,1)$. Let $F^{K S}(A)=(t, t)$. Note that $t>0$. Consider the following bargaining problems:

$$
S=\operatorname{comp}\{(t, 1),(t, t),(1, t)\}, \quad T=\operatorname{comp}\{(0,1),(t, t),(1,0)\}
$$

Note that $S, T \in \Sigma, T \subseteq A \subseteq S$. (SS) and (WE) imply that $F(S)=F(T)=\{(t, t)\}$. By (WMON), it then follows easily that $F(A)=\{(t, t)\}$. Thus, $F=F^{K S}$.

\section{$7 \quad$ Egalitarian solution}

Like the Kalai-Smorodinsky solution, the egalitarian solution to nonconvex bargaiging problems is also single-valued. In what follows, we summarize a characterization result obtained in $\mathrm{Xu}$ and Yoshihara (2006).

Theorem 16: A bargaining solution $F$ over $\Sigma$ is the egalitarian solution if and only if it satisfies (WE), (SS) and (CI).

Sketch of the proof. We illustrate the main idea behind a formal proof (see Xu and Yoshihara, 2006) with $n=2$.

Consider $A \in \Sigma$. Let $a=\left(a_{1}, a_{2}\right) \in A$ be weakly efficient and $a_{1}=a_{2}$. Let $m=$ $\max \left\{m_{1}(A), m_{2}(A)\right\}$. Note that $m>0$. Consider the bargaining problem $B=\operatorname{comp}\left\{\left(m, a_{2}\right),\left(a_{1}, m\right)\right\}$. Note that $B$ is symmetric and $\left(a_{1}, a_{2}\right)$ is weakly efficient in $B$. By (WE) and (SS), it then follows that $\{a\}=F(B)$. Note that $A \subseteq B$. By $(\mathrm{CI}), F(A)=\{a\}$.

It may be noted that both the Kalai-Smorodinsky solution and the egalitarian solution satisfy (WE) and (SS). Their differences lie in the fact that the Kalai-Smorodinsky solution satisfies (SI) and (WCI), which is formally weaker than (CI), but fails to satisfy (CI), while the egalitarian solution satisfies (CI) but fails to satisfy (SI).

To conclude our discussion on the egalitarian solution, we note that Conley and Wilkie (1991) show Kalai's (1977) characterization of the egalitarian solution continues to hold on the domain of nonconvex bargaining problems.

\section{Economic Applications}

In this section, we discuss two interesting applications of nonconvex bargaining theories to economics, in particular, welfare economics. The first application is about the debate of distributive justice between equality of welfare and equality of resources, and the second application is concerned with equality of opportunity sets. 


\subsection{Bargaining theory and Distributive Justice}

In this subsection, we consider the classical resource allocation problems in economic environments, where economic environments (hereafter, economies) are pure exchange economies or production economies, and define allocation rules, each of which maps an economy to a subset of feasible resource allocations in the economy. In this context, a bargaining solution is formulated as an allocation rule in the following way: first, from each economy, a utility possibility set is uniquely derived and this utility possibility set is regarded as a bargaining problem; secondly, for each economy, a set of optimal allocations is specified by an allocation rule and, corresponding to the specified optimal allocations, their counterparts of utility allocations are regarded as the recommendation by a bargaining solution. In the standard class of economies, the corresponding utility possibility set is compact, comprehensive, and nonconvex, where the last property follows if the admissible class of utility functions are quasi-concave. Therefore, the bargaining problems derived from the classical economic resource allocations are generally nonconvex. Thus, the nonconvex bargaining theory can be applied to such allocation problems. In the following, we will survey the axiomatic studies of nonconvex bargaining solutions which are motivated by the contemporary theories of distributive justice, such as Dworkin's (1981b) theory of equality of resources.

Dworkin's (1981b) theory of equality of resources says that an individual should be responsible for his less-preferred situation if such a situation is caused by his free will as represented by his own utility function, but he should not be held responsible if his situation is due to his bad luck such as handicaps and/or low skill. It then goes on to argue that an individual should be compensated by the society if his unpleasant state is attributed to his bad luck, but should not be compensated if it is caused by his own utility function such as "expensive tastes." The following subsection presents an economic model that incorporates this argument into the classical resource allocation problems.

\subsubsection{Economic environments}

There are infinitely many types of goods (commodities). The universe of "potential goods" is denoted by $\Xi$, and the class of non-empty and finite subsets of $\Xi$ is designated by $\mathcal{M}$, with generic elements, $K, L, M, \ldots$, each is to be called a finite list of commodities. The cardinality of $M \in \mathcal{M}$ is denoted by $\# M=m$. For each $M \in \mathcal{M}$, let us denote a generic commodity bundle in $\mathbb{R}_{+}^{\# M}$ by $x$. To abuse the notation slightly, we shall write $\mathbb{R}_{+}^{\# M}$ as $\mathbb{R}_{+}^{m}$.

Given $M \in \mathcal{M}$, one technology that can produce up to $M$-goods is described by a production possibility set $Y \subseteq \mathbb{R}_{+} \times \mathbb{R}_{+}^{m}$, where it is assumed that:

\section{A.1 $0 \in Y$.}

A.2 $Y$ is closed, convex, and comprehensive.

The universal set of such production possibility sets which produce up to $M$-goods is denoted by $\mathcal{Y}^{M}$. Let $\mathcal{Y} \equiv \underset{M \in \mathcal{M}}{\cup} \mathcal{Y}^{M}$. Let $\partial Y \equiv\left\{(x, y) \in Y \mid \nexists\left(x^{\prime}, y^{\prime}\right) \in Y\right.$ s.t. $\left.\left(-x^{\prime}, y^{\prime}\right) \gg(-x, y)\right\}$. Note that a special case of production possibility sets is $Y=\mathbb{R}_{+} \times\left\{\omega_{M}\right\}$, where $\omega_{M} \in \mathbb{R}_{++}^{m}$ is a fixed positive vector of commodities. This kind of production set corresponds to a pure exchange economy, as the society can access to the commodities $\omega_{M}$ regardless of the amount 
of labor being used. In the following discussion, we will use $\omega_{M}$ to represent such a type of specific "production set."

By assuming one type of labor, it follows that every individual's labor is homogeneous, although their labor skills are possibly unequal. The population in the economy is given by the set $N=\{1, \cdots, n\}$, where $2 \leq n<+\infty$. Assume that all individuals have the same upper bound of labor time $\bar{x}, 0<\bar{x}<+\infty$. Each individual $i$ is characterized by a labor skill which is represented by a non-negative real number, $s_{i} \in \mathbb{R}_{+}$. The number $s_{i}$ indicates $i$ 's labor supply per hour measured in efficiency units. Thus, if $x_{i}$ is the labor time expended by $i$, then his labor supply in efficiency units is $s_{i} x_{i}$. Let us denote the universal set of labor skills for all individuals by $\mathcal{S} \subseteq \mathbb{R}_{+}$.

Each individual is also characterized by his own utility function defined over a consumption space. The consumption space is given by $[0, \bar{x}] \times \mathbb{R}_{+}^{m}$ whenever there exist $m$ types of commodities that are able to be produced in the society. Let $\mathcal{U}^{M}$ be the set of all (realvalued) quasi-concave and continuous utility functions defined on $[0, \bar{x}] \times \mathbb{R}_{+}^{m}$, such that any $u \in \mathcal{U}^{M}$ is non-increasing in $[0, \bar{x}]$, non-decreasing in $\mathbb{R}_{+}^{m}, u(0, \mathbf{0})=u(\bar{x}, \mathbf{0})=0$, and for all $(x, y) \in[0, \bar{x}] \times \mathbb{R}_{+}^{m}, \lim _{t \rightarrow \infty}(1 / t) \cdot u(x, t y)=0$.

Given $M \in \mathcal{M}$, an economy with $M$-commodities is described by a list $\mathbf{e}=(M, \mathbf{u}, \mathbf{s}, Y)=$ $\left(M,\left(u_{i}\right)_{i \in N},\left(s_{i}\right)_{i \in N}, Y\right)$, where $M \in \mathcal{M}, \mathbf{u} \in \mathcal{U}^{M n}, \mathbf{s} \in \mathcal{S}^{n}, Y \in \mathcal{Y}^{M}$, and $\mathcal{U}^{M n}$ and $\mathcal{S}^{n}$ stand, respectively, for the $n$-fold Cartesian product of $\mathcal{U}^{M}$ and that of $\mathcal{S}$. Let $\mathcal{E}^{M}$ be the class of all such economies with $M$-goods. Let $\mathcal{E} \equiv \underset{M \in \mathcal{M}}{\cup} \mathcal{E}^{M}$. Note that this definition of economies implies that the change in the dimension of consumption space is caused by the change in production technology, while it may also induce the change of individuals' utility functions.

Given $\mathbf{e}=(M, \mathbf{u}, \mathbf{s}, Y) \in \mathcal{E}^{M}$, a vector $\mathbf{z}=\left(z_{i}\right)_{i \in N} \in\left([0, \bar{x}] \times \mathbb{R}_{+}^{m}\right)^{n}$ constitutes a feasible allocation for $\mathbf{e} \in \mathcal{E}^{M}$ if for all $i \in N, z_{i}=\left(x_{i}, y_{i}\right)$, and $\left(\sum s_{i} x_{i}, \sum y_{i}\right) \in Y$. Since the profiles of labor time and of skills are respectively $\left(x_{i}\right)_{i \in N}$ and $\left(s_{i}\right)_{i \in N}$, the aggregate amount of labor input in efficiency units is $\sum s_{i} x_{i}$, which is transformed into $M$-commodities through the production possibility set $Y$. We denote by $Z(\mathbf{e})$ the set of feasible allocations for $\mathbf{e} \in \mathcal{E}^{M}$. Let $Z(\mathcal{E}) \equiv \cup_{\mathbf{e} \in \mathcal{E}} Z(\mathbf{e})$.

Given $\mathbf{e}=(M, \mathbf{u}, \mathbf{s}, Y) \in \mathcal{E}$, the utility possibility set of $\mathbf{e} \in \mathcal{E}$ is:

$$
A(\mathbf{e}) \equiv\left\{\overline{\mathbf{u}}=\left(\bar{u}_{i}\right)_{i \in N} \in \mathbb{R}_{+}^{n} \mid \exists \mathbf{z}=\left(z_{i}\right)_{i \in N} \in Z(\mathbf{e}), \forall i \in N, \bar{u}_{i}=u_{i}\left(z_{i}\right)\right\} .
$$

Note that the utility possibility set $A(\mathbf{e})$ is a compact and comprehensive set in $\mathbb{R}_{+}^{n}$ containing the origin. Let $\Sigma \equiv\left\{A \subseteq \mathbb{R}_{+}^{n} \mid \exists \mathbf{e} \in \mathcal{E}, A=A(\mathbf{e})\right\}$ be the class of all such utility possibility sets, which constitutes the available class of nonconvex (normalized) bargaining problems.

An allocation rule is a correspondence $\varphi: \mathcal{E} \rightarrow Z(\mathcal{E})$ which associates to each $\mathbf{e}=$ $(M, \mathbf{u}, \mathbf{s}, Y) \in \mathcal{E}$, a non-empty subset $\varphi(\mathbf{e})$ of $Z(\mathbf{e})$. The allocation rule $\varphi$ is assumed to be essentially a function; that is, for all $\mathbf{e}=(M, \mathbf{u}, \mathbf{s}, Y) \in \mathcal{E}$, if $\mathbf{z} \in \varphi(\mathbf{e})$ and $\mathbf{z}^{\prime} \in \varphi(\mathbf{e})$, then $\mathbf{u}(\mathbf{z})=\mathbf{u}\left(\mathbf{z}^{\prime}\right)$, where $\mathbf{u}(\mathbf{z})=\left(u_{i}\left(z_{i}\right)\right)_{i \in N}$ and $\mathbf{u}\left(\mathbf{z}^{\prime}\right)=\left(u_{i}\left(z_{i}^{\prime}\right)\right)_{i \in N}$. Moreover, $\varphi$ is assumed to be a full correspondence; that is, for all $\mathbf{e}=(M, \mathbf{u}, \mathbf{s}, Y) \in \mathcal{E}$, if $\mathbf{z} \in \varphi(\mathbf{e}), \mathbf{z}^{\prime} \in Z(\mathbf{e})$, and $\mathbf{u}(\mathbf{z})=\mathbf{u}\left(\mathbf{z}^{\prime}\right)$, then $\mathbf{z}^{\prime} \in \varphi(\mathbf{e})$. The allocation rule $\varphi$ attains a bargaining solution $F$ if for all $\mathbf{e}=(M, \mathbf{u}, \mathbf{s}, Y) \in \mathcal{E}, \mu_{\varphi}(\mathbf{e})=F(S(\mathbf{e}))$, where $\mu_{\varphi}(\mathbf{e}) \equiv \mathbf{u}(\varphi(\mathbf{e}))$. Denote the class of all the allocation rules, each of which attains some bargaining solution, by $\Phi_{\mathcal{F}}$. 


\subsubsection{Axioms using Economic Information}

First, the domain assumption on allocation rules is:

Axiom $D^{\mathcal{E}}$ : The allocation rule $\varphi$ is a full correspondence which is essentially a function and is defined on the class of economies $\mathcal{E}$.

The following are well-known axioms on allocation rules:

Welfarism (W) [Roemer (1988)]: For all $\mathbf{e}=(M, \mathbf{u}, \mathbf{s}, Y), \mathbf{e}^{\prime}=\left(M^{\prime}, \mathbf{u}^{\prime}, \mathbf{s}^{\prime}, Y^{\prime}\right) \in \mathcal{E}$, if $S(\mathbf{e})=S\left(\mathbf{e}^{\prime}\right)$, then $\mu_{\varphi}(\mathbf{e})=\mu_{\varphi}\left(\mathbf{e}^{\prime}\right)$.

Weak Pareto Efficiency (WPE): For all $\mathbf{e}=(M, \mathbf{u}, \mathbf{s}, Y) \in \mathcal{E}$ and $\mathbf{z} \in \varphi(\mathbf{e})$, there is no feasible allocation $z^{\prime} \in Z(e)$ such that $u_{i}\left(z_{i}^{\prime}\right)>u_{i}\left(z_{i}\right)$ for all $i \in N$.

Weak Equal Treatment of Equals (WETE) [Fleurbaey and Maniquet (1999)]: For all $\mathbf{e}=(M, \mathbf{u}, \mathbf{s}, Y) \in \mathcal{E}$, if $u_{i}=u_{j}$ and $s_{i}=s_{j}$ for all $i, j \in N$, then for all $\mathbf{z} \in \varphi(\mathbf{e})$, $u_{i}\left(z_{i}\right)=u_{j}\left(z_{j}\right)$ for all $i, j \in N$.

Note that all allocation rules in $\Phi_{\mathcal{F}}$ satisfy Axiom $D^{\mathcal{E}}$ and $(\mathbf{W})$.

The next axiom is to stipulate the performance of allocation rules in the case of particular types of technological changes. It requires coherent treatments of allocation problems between before and after technological innovations if such innovations make it possible to produce new commodities which nobody wants to consume: Given $(x, y) \in[0, \bar{x}] \times \mathbb{R}_{+}^{m}$ and $u_{i} \in \mathcal{U}^{M}$, let there be $K \subsetneq M$ such that for all $y_{K}^{\prime} \equiv\left(y_{f}^{\prime}\right)_{f \in K} \in \mathbb{R}_{+}^{k}, u_{i}\left(x, y_{K}^{\prime}, y_{M \backslash K}\right)=$ $u_{i}\left(x, y_{K}, y_{M \backslash K}\right)$, where $y_{K} \equiv\left(y_{f}\right)_{f \in K}$. Then, we say that agent $i \in N$ is indifferent to each good of $K \subsetneq M$ at $(x, y)$. Given $Y \in \mathcal{Y}^{M}$ and $K \subsetneq M$, let

$$
P_{M \backslash K}(Y) \equiv\left\{\left(x, y_{M \backslash K}\right) \in \mathbb{R}_{+} \times \mathbb{R}_{+}^{m-k} \mid \exists y_{K} \in \mathbb{R}_{+}^{k}:\left(x, y_{K}, y_{M \backslash K}\right) \in Y\right\} .
$$

Consistency w.r.t. Technological Innovation (CTI) [Yoshihara (2003)]: Let e = $(M, \mathbf{u}, \mathbf{s}, Y) \in \mathcal{E}^{M}$, and let $\widehat{\mathbf{z}}=\left(\widehat{x}_{i}, \widehat{y}_{M i}\right)_{i \in N} \in \varphi(\mathbf{e})$ be weakly Pareto efficient. ${ }^{5}$ Let $\mathbf{e}^{\prime}=$ $\left(M \cup L, \mathbf{u}^{\prime}, \mathbf{s}, Y^{\prime}\right) \in \mathcal{E}^{M \cup L}$, where $M \cap L=\varnothing$, be such that (1) $P_{M}\left(Y^{\prime}\right)=Y$, (2) for any $\mathbf{z}=\left(x_{i}, y_{M i}\right)_{i \in N} \in W P E(\mathbf{e})$, there exists $\left(y_{L i}(\mathbf{z})\right)_{i \in N} \in \mathbb{R}_{+}^{n l}$ such that

$$
u_{i}^{\prime}\left(x_{i}, y_{M i}, y_{L i}(\mathbf{z})\right)=u_{i}\left(x_{i}, y_{M i}\right)(\forall i \in N) \text { and }\left(x_{i}, y_{M i}, y_{L i}(\mathbf{z})\right)_{i \in N} \in W P E\left(\mathbf{e}^{\prime}\right)
$$

and (3) every agent $i \in N$ is indifferent to each good of $L$ at $\left(\widehat{x}_{i}, \widehat{y}_{M i}, \mathbf{0}\right)$. Then, $\left(\widehat{x}_{i}, \widehat{y}_{M i}, \mathbf{0}\right)_{i \in N} \in$ $\varphi\left(\mathbf{e}^{\prime}\right)$.

A motivation for (CTI) is presented as follows. Let, in an economy e with $M$-commodities, $\widehat{\mathbf{z}}=\left(\widehat{x}_{i}, \widehat{y}_{M i}\right)_{i \in N}$ be a recommendation by the allocation rule $\varphi$, and be a (weakly) Pareto efficient allocation. Next, let the economy change from $\mathbf{e} \in \mathcal{E}^{M}$ to $\mathbf{e}^{\prime} \in \mathcal{E}^{M \cup L}$, where the economy $\mathbf{e}^{\prime}$ inherits from $\mathbf{e}$ the features of the agents' utility functions on $M$-commodities in the intimate way that the condition (2) of (CTI) stipulates: for every weakly Pareto efficient

\footnotetext{
${ }^{5}$ When we discuss dimensional changes in consumption spaces, we often denote individual $i^{\prime}$ s consumption vector of $M$-goods by $y_{M i} \in \mathbb{R}_{+}^{m}$.
} 
allocation $\mathbf{z}$ in $\mathbf{e}$, there exists an allocation $\left(y_{L i}(\mathbf{z})\right)_{i \in N}$ of $L$-commodities such that the new allocation, $\left(x_{i}, y_{M i}, y_{L i}(\mathbf{z})\right)_{i \in N}$, which is obtained by combining $\mathbf{z}$ and $\left(y_{L i}(\mathbf{z})\right)_{i \in N}$, achieves the same utility allocation in $\mathbf{e}^{\prime}$ as the utility allocation derived from $\mathbf{z}$ in $\mathbf{e}$. Condition (1) stipulates the feature of technological change from $Y$ to $Y^{\prime}$ which makes the transition from the economy $\mathbf{e}$ to $\mathbf{e}^{\prime}$ in the way that while the production ability of $M$-commodities is preserved, the new commodities $L$ can be produced in the new economy. However, all agents are indifferent to the consumption of these commodities as condition (3) specifies. Given such a transition, (CTI) requires that every agent in the new economy should be guaranteed at least his/her welfare level enjoyed in the original economy, as no individuals want to consume $L$-commodities, and any other components in the economy e and the economy $\mathbf{e}^{\prime}$ are essentially identical.

Remark 2: The axiom (W) implies (CTI) under Axiom $D^{\mathcal{E}}$, but not the converse, even if $\varphi$ obeys Axiom $D^{\mathcal{E}}$.

Roemer (1986, 1988) also introduces a similar axiom to (CTI), which is defined in the class of pure exchange economies ${ }^{6}$ and named Consistency of Resource Allocation across Dimension (CONRAD). Like (CTI), the axiom (CONRAD) also requires coherent treatments of allocation problems when the dimension of commodities available in economies changes. It considers a situation that an economy with a social endowment of $m+l$ dimensional vector of commodities, $\left(\omega_{M}, \omega_{L}\right) \in \mathbb{R}_{++}^{m+l}$, changes to an economy with that of $m$ dimensional commodity vector, $\omega_{M} \in \mathbb{R}_{++}^{m}$. Accordingly, the agents' utility functions in the $m$ dimensional economy is essentially 'inherited' from those in the $m+l$ dimensional economy, which is similar to the condition (2) of (CTI). Then, if $\left(y_{M i}, y_{L i}\right)_{i \in N}$ is $\varphi$-optimal in the first economy, then (CONRAD) requires that $\left(y_{M i}\right)_{i \in N}$ is $\varphi$-optimal in the second economy.

Let $\mathcal{E}_{p e}$ be the set of pure exchange economies with generic element $\mathbf{e}=\left(M, \mathbf{u}, \mathbf{0}, \omega_{M}\right)$. Then, the next axiom is introduced by Roemer (1986a) to consider allocation problems in pure exchange economies.

Resource Monotonicity (RMON) [Roemer (1986a)]: For all $\mathbf{e}=\left(M, \mathbf{u}, \mathbf{0}, \omega_{M}\right), \mathbf{e}^{\prime}=$ $\left(M, \mathbf{u}, \mathbf{0}, \omega_{M}^{\prime}\right) \in \mathcal{E}_{p e}$ such that $\omega_{M} \leq \omega_{M}^{\prime}$, and all $\mathbf{z} \in \varphi(\mathbf{e})$ and all $\mathbf{z}^{\prime} \in \varphi\left(\mathbf{e}^{\prime}\right), u_{i}\left(z_{i}\right) \leq u_{i}\left(z_{i}^{\prime}\right)$ for all $i \in N$.

\subsubsection{Axiom on Responsibility}

Given $M \in \mathcal{M}$, note that for any utility function $u \in \mathcal{U}^{M}$, there is a utility-unit $b^{u} \in \mathbb{R}_{+}$, by which the level of utility assigned by the function $u$ is measured: that is, if $u(z)=b^{u}$ for some $z$, it implies that the level of utility $u(z)$ is just "one." Then, for each utility-unit $b^{u}$, there is a corresponding set $B(u) \subsetneq[0, \bar{x}] \times \mathbb{R}_{+}^{m}$ of base-consumption for $u$ such that for all $z \in B(u), u(z)=b^{u}$.

Now, let us take any two utility functions $u, u^{\prime} \in \mathcal{U}^{M}$ for which there is a positive scalar $\lambda>0$ such that $u^{\prime}=\lambda \cdot u$. If $\lambda=\frac{b^{u^{\prime}}}{b^{u}}$, then $u^{\prime}$ is just obtained by a change in utility-units from

\footnotetext{
${ }^{6}$ An economy $\mathbf{e}=(M, \mathbf{u}, \mathbf{s}, Y) \in \mathcal{E}$ is called a pure exchange economy if and only if $\mathbf{s}=\mathbf{0}$ and there exists a social endowment vector $\omega_{M} \in \mathbb{R}_{++}^{m}$ of commodities such that $Y=\mathbb{R}_{+} \times\left\{\omega_{M}\right\}$. In the following, we will use the notation $\left(M, \mathbf{u}, \mathbf{0}, \omega_{M}\right)$ to denote a generic element of pure exchange economies.
} 
$b^{u}$ to $b^{u^{\prime}}$, so that $u$ and $u^{\prime}$ are essentially the same utility representation. In this case, note that $B(u)=B\left(u^{\prime}\right)$. In contrast, if $b^{u}=b^{u^{\prime}}$, then the change from $u$ to $u^{\prime}$ can be explained not by the change in utility-units, but rather by a change in utility intensity. Note that if the change from $u$ to $u^{\prime}$ comes from the change in utility intensity, then we have $B(u) \neq B\left(u^{\prime}\right)$ and $B(u) \cap B\left(u^{\prime}\right)=\varnothing$.

One typical example of the above change in utility intensity is the case of individual development of "expensive taste," which was discussed by Dworkin (1981a). Consider a case in which an individual develops his expensive taste, so that even if his underlying preference ordering and his risk attitude are invariant, he can no longer enjoy the same level of welfare as he did before developing his expensive taste, without receiving a larger consumption vector than before. ${ }^{7}$ This case is simply formulated as a process of a linear transformation of a utility function via a change in utility intensity.

Given $M \in \mathcal{M}$ and $\mathbf{u} \in \mathcal{U}^{M n}$, let $\mathbf{b}^{\mathbf{u}} \equiv\left(b^{u_{i}}\right)_{i \in N}$. Then:

Independence of Utility Intensities (IUI) [Yoshihara (2003)]: For all $\mathbf{e}=(M, \mathbf{u}, \mathbf{s}, Y), \mathbf{e}^{\prime}=$ $\left(M, \mathbf{u}^{\prime}, \mathbf{s}, Y\right) \in \mathcal{E}$ with $\mathbf{b}^{\mathbf{u}}=\mathbf{b}^{\mathbf{u}^{\prime}}$, if there exists a vector $\mathbf{a}=\left(a_{i}\right)_{i \in N} \in \mathbb{R}_{++}^{n}$ such that $u_{i}^{\prime}=a_{i} \cdot u_{i}$ for all $i \in N$, then $\varphi(\mathbf{e})=\varphi\left(\mathbf{e}^{\prime}\right)$.

In production economies with differences in production skills among agents, according to the Dworkinian theory of equality of resources, the change in utility intensity of any agent is not a subject for social compensation, but a matter of personal responsibility. So, the axiom (IUI) requires that the allocation rule should not take into account such a change to determine resource allocations. ${ }^{8}$

\subsubsection{Axioms on Compensation}

The next two axioms are relevant to compensation for low skills:

Skill Monotonicity (SM) [Fleurbaey and Maniquet (1999)]: For all $\mathbf{e}=(M, \mathbf{u}, \mathbf{s}, Y), \mathbf{e}^{\prime}=$ $\left(M, \mathbf{u}, \mathbf{s}^{\prime}, Y\right) \in \mathcal{E}$ such that $\mathbf{s} \leq \mathbf{s}^{\prime}$, and all $\mathbf{z} \in \varphi(\mathbf{e})$ and $\mathbf{z}^{\prime} \in \varphi\left(\mathbf{e}^{\prime}\right), u_{i}\left(z_{i}\right) \leq u_{i}\left(z_{i}^{\prime}\right)$ for all $i \in N$.

Independence of Skill Endowments (ISE) [Yoshihara (2003)]: Let $\mathbf{e}=(M, \mathbf{u}, \mathbf{s}, Y), \mathbf{e}^{\prime}=$ $\left(M, \mathbf{u}, \mathbf{s}^{\prime}, Y\right) \in \mathcal{E}$ be such that $Z(\mathbf{e})=Z\left(\mathbf{e}^{\prime}\right)$. Then, $\varphi(\mathbf{e})=\varphi\left(\mathbf{e}^{\prime}\right)$.

Among these two axioms, (SM) is strong enough to keep the implication of compensation for low skill, while (ISE) is a rather weak requirement as an axiom of compensation.

A weaker version of $(\mathbf{S M})$ is also introduced. Given $\mathbf{e}=(M, \mathbf{u}, \mathbf{s}, Y) \in \mathcal{E}$ and $i \in N$, let

$$
m^{i}(Z(\mathbf{e})) \equiv\left\{z_{i}^{\prime} \in[0, \bar{x}] \times \mathbb{R}_{+}^{m} \mid z_{i}^{\prime}=\underset{\mathbf{z} \in Z(\mathbf{e}), z_{i} \text { is } i \text {-th component of } \mathbf{z}}{\arg \max } u_{i}\left(z_{i}\right)\right\} .
$$

\footnotetext{
${ }^{7}$ In this explanation, it is not necessary to assume interpersonal comparability of utilities. The notion of change in utility intensity only presumes intrapersonal comparison of utilities.

${ }^{8}$ As discussed above, the situation that someone's utility intensity decreases can be interpreted as the development of an "expensive taste." Then, IUI requires that this person should not be compensated by the allocation rule for his decrease of utility-productivity due to his developed expensive taste.
} 
Then:

Weak Skill Monotonicity (WSM) [Yoshihara (2006)]: Let $\mathbf{e}=(M, \mathbf{u}, \mathbf{s}, Y), \mathbf{e}^{\prime}=\left(M, \mathbf{u}, \mathbf{s}^{\prime}, Y\right) \in$ $\mathcal{E}$ be such that $\mathbf{s} \leq \mathbf{s}^{\prime}$, and $m^{i}(Z(\mathbf{e})) \cap m^{i}\left(Z\left(\mathbf{e}^{\prime}\right)\right) \neq \varnothing$ for all $i \in N$. Then, for all $\mathbf{z} \in \varphi(\mathbf{e})$ and all $\mathbf{z}^{\prime} \in \varphi\left(\mathbf{e}^{\prime}\right)$, we have $u_{i}\left(z_{i}\right) \leq u_{i}\left(z_{i}^{\prime}\right)$ for all $i \in N$.

This axiom requires that if the profile of skills is improved from $\mathbf{s}$ to $\mathbf{s}^{\prime}$, but everyone's ideal utility point remains invariant, then no individual should be made worse off by this environmental improvement. The presumption of this axiom is non-vacuous. To see it, let $\mathbf{z} \in Z(\mathbf{e})$ and $\mathbf{z}^{\prime} \in Z\left(\mathbf{e}^{\prime}\right)$ be such that $z_{i} \in m^{i}(Z(\mathbf{e})), z_{i}^{\prime} \in m^{i}\left(Z\left(\mathbf{e}^{\prime}\right)\right), y_{i} \leq y_{i}^{\prime}$ and $x_{i}=x_{i}^{\prime}$ for each $i \in N$, due to $\mathbf{s} \leq \mathbf{s}^{\prime}$. Then, if $u_{i}$ is satiated at $z_{i}$, then $z_{i} \in m^{i}(Z(\mathbf{e})) \cap m^{i}\left(Z\left(\mathbf{e}^{\prime}\right)\right)$ can hold for each $i \in N$.

\subsubsection{Bargaining Allocation Rules and their Characterizations}

Here, the following two bargaining allocation rules will be discussed in terms of the theory of equality of resources:

Definition 5: An allocation rule $\varphi^{E}$ is the egalitarian rule if it attains the egalitarian solution: for all $\mathbf{e} \in \mathcal{E}, \mu_{\varphi^{E}}(\mathbf{e})=F^{E}(A(\mathbf{e}))$.

Definition 6: An allocation rule $\varphi^{K S}$ is the Kalai-Smorodinsky rule if it attains the KalaiSmorodinsky solution: for all $\mathbf{e} \in \mathcal{E}, \mu_{\varphi^{K S}}(\mathbf{e})=F^{K S}(A(\mathbf{e}))$.

In the literature of bargaining theory on economic environments, an allocation rule which satisfies Axiom $D^{\mathcal{E}}$ and attains the Nash bargaining solution is also defined as the Nash allocation rule. Such an allocation rule is well-defined whenever all available utility functions are restricted to be concave, and so all bargaining problems derived from such economies are convex. However, here we allow that utility functions are quasi-concave but not necessarily concave, and so the derived bargaining problems could be nonconvex. In such a case, the allocation rule which attains the Nash solution (Definition 1 of this paper) does not satisfy Axiom $D^{\mathcal{E}}$, as the Nash allocation rule is not essentially a function.

First, let us consider allocation problems in pure exchange economies. Then, according to Roemer (1986), the theory of equality of resources could be formulated by means of (WPE), (WETE), (RMON), and (CONRAD). Moreover, it can be shown that an allocation rule is egalitarian if and only if it satisfies Axiom $D^{\mathcal{E}}$, (WPE), (WETE), (RMON), and (CONRAD). From this analysis, Roemer (1986) concludes that equality of resources logically implies equality of welfare, since the egalitarian rule is the mathematical representation of the theory of equality of welfare. This implies a criticism against Dworkin's (1981b) theory of equality of resources.

However, it was questioned whether equality of resources is properly characterized by the axioms mentioned above. Indeed, these axioms do not reflect any claim on responsibility and compensation that Dworkin (1981b) regards as the core principles of the theory of equality of resources. Therefore, the logical relationship between equality of welfare and equality of resources claimed by Roemer (1986) would not be valid if equality of resources is 
characterized by the principles of responsibility and compensation, rather than by the above four axioms.

Given this conceptual debate, Yoshihara (2003, 2006) examines allocation problems in production economies with different skills by using the axiom of responsibility, (IUI), and the axioms of compensation for low skills, (SM) and its weaker variants.

In this case, an allocation rule would be the first best from the viewpoint of Dworkin's (1981b) theory of equality of resources if it satisfies (IUI) and (SM) together with weaker requirements of efficiency and fairness. However, there is no efficient bargaining allocation rule which satisfies both the axiom of responsibility and the stronger axiom of compensation:

Theorem 17 [Yoshihara (2003)]: There is no allocation rule in $\Phi_{\mathcal{F}}$ which satisfies (WPE), (WETE), (IUI), and (SM).

Given the impossibility of the first best solution of equality of resources, one second best resolution shown by Yoshihara (2003) is to remove the responsibility axiom (IUI), while keeping the compensation axiom (SM), which results in the egalitarian solution.

Theorem 18 [Yoshihara (2003)]: The allocation rule $\varphi$ satisfies $D^{\mathcal{E}}$, (WPE), (WETE), (SM), (ISE), and (CTI) if and only if $\varphi=\varphi^{E}$.

Within the list of axioms used in the above characterization, the axioms $D^{\mathcal{E}}$, (ISE), and (CTI) are logically necessary for all bargaining allocation rules in $\Phi_{\mathcal{F}}$. Moreover, (WPE) and (WETE) are minimally necessary for ethically acceptable bargaining allocation rules. Therefore, it is the axiom (SM) which uniquely characterizes the egalitarian rule among any ethically acceptable bargaining allocation rules.

Another second best resolution is to weaken the compensation axiom from (SM) to (WSM), while preserving (IUI), which results in the KS allocation rule:

Theorem 19 [Yoshihara (2006)]: The allocation rule $\varphi$ satisfies $D^{\mathcal{E}}$, (WPE), (WETE), (WSM), (IUI), ISE, and (CTI) if and only if $\varphi=\varphi^{K S}$.

As in the discussion after the presentation of Theorem 18, we can say that the axioms (IUI) and (WSM) uniquely characterize the KS allocation rule within the class of all ethically acceptable bargaining allocation rules.

In this way, the well-known solutions to nonconvex bargaining problems can be characterized from the viewpoint of equality of resources à la Dworkin (1981b) by reformulating the classical bargaining problems as the standard resource allocation problems in economic environments. In addition to Roemer $(1986,1988)$ and Yoshihara $(2003,2006)$ introduced in this subsection, Ginés and Marhuenda (2000) and Chen and Maskin (1999) also respectively provide an axiomatic characterization of the egalitarian solution by considering the economic models with finite dimensional commodity spaces. In these works, the egalitarian solution is characterized by means of Pareto efficiency, weak symmetry, and payoff monotonicity. Weak symmetry is essentially the same as the (WETE) axiom, while payoff monotonicity can be summarized as follows: if everyone's utility-productivity is improved, then everyone's utility in the bargaining outcome should be improved. Obviously, payoff monotonicity cannot be justified by either Roemer's (1986) axiomatic formulation of equality of resources or the 
principles of responsibility and compensation. Thus, though the characterization result by Ginés and Marhuenda (2000) and Chen and Maskin (1999) is elegant, it has no implication of the equality of resources, unlike Roemer $(1986,1988)$ and Yoshihara $(2003,2006)$.

\subsection{Bargaining over opportunity sets}

In this subsection, we again consider economic resource allocation problems in pure exchange economies, but we also introduce each agent's opportunity set, which is interpreted as a set of feasible options or alternatives available to the individual for living by using commodities allocated to him. Those alternatives can be interpreted broadly as commodity bundles, or bundles of characteristics à la Lancaster and Gorman, or bundles of functionings à la Sen (1980, 1985), and Nussbaum $(1988,1993,2000)$. A resource allocation of commodities in a pure exchange economy then identifies a collection of opportunity sets, one for each individual in the economy.

In this context, we define a bargaining problem on assignments of opportunity sets, in which a bargaining problem is the set of all available assignments of opportunity sets among agents. Correspondingly, a bargaining solution maps each of such bargaining problems to an assignment of individual opportunity sets.

There are actual examples of bargaining problems in which each recommendation by the "fair arbitrator" is made not based on utilities of the individuals involved, but on opportunity sets that the recommended resource allocation of commodities may give rise to the involved individuals. For example, when educational resources are to be allocated among local public schools, the local government's board of education, as the "fair arbitrator," may propose an allocation that "equalizes" school children's opportunity sets for future jobs, skills, college admissions, lives, etc. A bargaining theory addressing such an example is well in line with the recent literature on opportunities and equality of opportunities, such as Sen $(1980,1985)$, Arneson (1989), and Cohen (1993) in political philosophy, and Sen (2002), Pattanaik and Xu (1990), Kranich (1996), and Herrero (1996) in welfare economics.

In this subsection, following Xu and Yoshihara (2019a), we will introduce the egalitarian solution to bargaining problems on assignments of opportunity sets as a recommendation based on the equality of opportunity principle. Then, we will discuss the axiomatic characterization of such a solution. In particular, we will see how the bargaining problems on assignments of opportunity sets can be interpreted as an interesting example of the standard nonconvex bargaining problems.

\subsubsection{Economic environments}

As discussed in section 8.1, a pure exchange economy has the following profile $\mathbf{e}=\left(M, \mathbf{u}, \mathbf{0}, \omega_{M}\right)$. In addition, let us assume that there are $k$ basic living conditions in the economy, which are relevant for all individuals for the purpose of describing their objective well-beings attainable by means of their consumption vectors. These basic living conditions can be interpreted broadly. For example, they can be skills that individuals can develop through education, or they can be occupations which individuals can engage in after the graduation at school. Or they can be characteristics of commodities in the sense of Gorman (1980) and Lancaster 
(1966), or they can be various functionings according to Sen (1980, 1985) and Nussbaum $(1988,1993,2000)$.

An achievement of living condition $f$, where $f=1,2, \cdots, k$, by individual $i$ is denoted by $b_{i f} \in \mathbb{R}_{+}$. Individual $i$ 's achievement of basic living conditions is then given by listing $b_{i f}: \mathbf{b}_{i}=\left(b_{i 1}, \cdots, b_{i k}\right) \in \mathbb{R}_{+}^{k}$. Two crucial factors determine the achievement of individual's basic living conditions: one is the amount of resources or commodities she can access for attaining these living conditions, and the other is the individual's ability to realize these living conditions by utilizing commodities. Given $M \in \mathcal{M}$ and each individual $i \in N$, the latter is formulated as $i$ 's opportunity correspondence $c_{i}^{m}: \mathbb{R}_{+}^{m} \rightarrow \mathbb{R}_{+}^{k}$ which associates to every commodity vector $y_{i} \in \mathbb{R}_{+}^{m}$ a non-empty subset $c_{i}^{m}\left(y_{i}\right)$ of $\mathbb{R}_{+}^{k}$. The intended interpretation is that $i$ is able to have access to each living-condition vector $\mathbf{b}_{i} \in c_{i}^{m}\left(y_{i}\right)$ by means of his commodity vector $y_{i}$. Each opportunity correspondence satisfies the following requirements:

(a) For all $y_{i}, y_{i}^{\prime} \in \mathbb{R}_{+}^{m}$ such that $y_{i} \leq y_{i}^{\prime}, c_{i}^{m}\left(y_{i}\right) \subseteq c_{i}^{m}\left(y_{i}^{\prime}\right)$ holds;

(b) For some $y_{i} \in \mathbb{R}_{+}^{m} \backslash\{\mathbf{0}\}, c_{i}^{m}\left(y_{i}\right) \cap \mathbb{R}_{++}^{k} \neq \varnothing$ holds, and $c_{i}^{m}(\mathbf{0})=\{\mathbf{0}\}$;

(c) For all $y_{i} \in \mathbb{R}_{+}^{m}, c_{i}^{m}\left(y_{i}\right)$ is compact and comprehensive in $\mathbb{R}_{+}^{k}$; and

(d) $c_{i}^{m}$ is continuous on $\mathbb{R}_{+}^{m}$ with respect to the Hausdorff topology.

Requirement (a) is a monotonicity property: more commodities generate "larger" opportunity sets. Requirement (b) essentially says that commodities are "desirable": they can help individuals in achieving positive levels of basic living conditions. Requirement (c) stipulates that any given commodity bundle generates a bounded opportunity set. Finally, requirement (d) says that "small" changes in commodity bundles lead to "small" changes in opportunity sets.

Let $\mathfrak{C}^{M}$ be the set of all possible opportunity correspondences defined on $\mathbb{R}_{+}^{m}$, which satisfy the above (a), (b), (c) and (d). A pure exchange economy $\mathbf{e}=\left(M, \mathbf{u}, \mathbf{0}, \omega_{M}\right)$ is now extended with a profile $\mathbf{c}^{m} \equiv\left(c_{i}^{m}\right)_{i \in N} \in \mathfrak{C}^{M n}$ of opportunity correspondences. In the following, without loss of generality, let us denote a pure exchange economy with a profile of opportunity correspondences (an economy, hereafter) by a list $\mathbf{e}=\left(M, \mathbf{u}, \mathbf{c}^{m}, \omega_{M}\right)$. Then, given an economy $\mathbf{e}=\left(M, \mathbf{u}, \mathbf{c}^{m}, \omega_{M}\right) \in \mathcal{E}$, a vector $\boldsymbol{y}=\left(y_{i}\right)_{i \in N} \in \mathbb{R}_{+}^{m n}$ is feasible for $\mathbf{e} \in \mathcal{E}$ if for all $i \in N, y_{i} \in \mathbb{R}_{+}^{m}$, and $\sum y_{i} \leq \omega_{M}$. We denote by $Z(\mathbf{e})$ the set of feasible allocations for $\mathbf{e} \in \mathcal{E}$. Let $Z(\mathcal{E}) \equiv \cup_{\mathbf{e} \in \mathcal{E}} Z(\mathbf{e})$.

For each individual $i \in N$, given $M \in \mathcal{M}$ and given $i$ 's consumption vector $y_{i}, c_{i}^{m}\left(y_{i}\right)$ generates an opportunity set $C_{i}=c_{i}^{m}\left(y_{i}\right)$ for $i$. An opportunity assignment is a list of $n$ opportunity sets one for each individual in the society. Given $\mathbf{e}=\left(M, \mathbf{u}, \mathbf{c}^{m}, \omega_{M}\right) \in \mathcal{E}$, the set of possible opportunity assignments for $\mathbf{e} \in \mathcal{E}$ is:

$$
\mathcal{C}(\mathbf{e}) \equiv\left\{\mathbf{C}=\left(C_{i}\right)_{i \in N} \subseteq \mathbb{R}_{+}^{k n} \mid \exists \boldsymbol{y}=\left(y_{i}\right)_{i \in N} \in Z(\mathbf{e}): C_{i}=c_{i}^{m}\left(y_{i}\right)(\forall i \in N)\right\} .
$$

Note that for any $\mathbf{e}=\left(M, \mathbf{u}, \mathbf{c}^{m}, \omega_{M}\right) \in \mathcal{E}$, any $\mathbf{C}=\left(C_{i}\right)_{i \in N} \in \mathcal{C}(\mathbf{e})$, and any $i \in N$, the opportunity $C_{i}$ is a compact, comprehensive set in $\mathbb{R}_{+}^{k}$ containing the origin. Let $\Sigma \equiv$ $\{\mathcal{C} \mid \exists \mathbf{e} \in \mathcal{E}: \mathcal{C}=\mathcal{C}(\mathbf{e})\}$ be the class of all such possible sets of opportunity assignments.

\subsubsection{Opportunity sets and their ranking}

Let $\mathcal{K}$ be the universal class of compact, comprehensive subsets in $\mathbb{R}_{+}^{k}$ containing the origin. Thus, $C \in \mathcal{K}$ implies that for any $M \in \mathcal{M}$, there exists $c^{m} \in \mathfrak{C}^{M}$ such that for some $y \in \mathbb{R}_{+}^{m}$, 
$c^{m}(y)=C$. Then, an objective measure of alternative opportunity sets is formalized as a binary relation $R \subseteq \mathcal{K} \times \mathcal{K}$, which is assumed to satisfy (i) reflexivity: [for all $C \in \mathcal{K}$, $(C, C) \in R$ ], (ii) completeness: [for all $C, C^{\prime} \in \mathcal{K},\left(C, C^{\prime}\right) \in R$ or $\left(C^{\prime}, C\right) \in R$ ], and (iii) transitivity: [for all $C, C^{\prime}, C^{\prime \prime} \in \mathcal{K}$, if $\left(C, C^{\prime}\right) \in R \&\left(C^{\prime}, C^{\prime \prime}\right) \in R$, then $\left(C, C^{\prime \prime}\right) \in R$ ]. Thus, $R$ is an ordering over $\mathcal{K}$. Note $P$ and $I$ are respectively the asymmetric and symmetric parts of $R$.

For all $C, C^{\prime} \in \mathcal{K}$, we write $C>C^{\prime}$ if for all $\mathbf{b}^{\prime} \in C^{\prime}$, there exists $\mathbf{b} \in C$ such that $\mathbf{b} \gg \mathbf{b}^{\prime}$. Given the comprehensiveness of opportunity sets in $\mathcal{K}$, when $C>C^{\prime}$, then $C^{\prime}$ is necessarily a proper subset of $C$.

As in the literature on ranking opportunity sets such as Gaertner and Xu (2006), Pattanaik and $\mathrm{Xu}(2000,2007)$, and $\mathrm{Xu}(2002,2004)$, the ordering $R$ on $\mathcal{K}$ is assumed to satisfy the following property:

Monotonicity: For all $C, C^{\prime} \in \mathcal{K}$, if $C \supseteq C^{\prime}$ then $\left(C, C^{\prime}\right) \in R$, and if $C>C^{\prime}$, then $\left(C, C^{\prime}\right) \in P$.

Representability: There exists a real-valued, continuous function $G: \mathcal{K} \rightarrow \mathbb{R}_{+}$such that for all $C, C^{\prime} \in \mathcal{K}$,

$$
G(C) \geq G\left(C^{\prime}\right) \Leftrightarrow\left(C, C^{\prime}\right) \in R
$$

\subsubsection{Bargaining problems on opportunity assignments}

Analogous to the standard bargaining model, here each $\mathcal{C} \in \Sigma$ is a bargaining problem on opportunity assignments, and $\Sigma$ is the domain of such bargaining problems. Moreover, a solution to such a problem is to pick up a subset of opportunity assignments $\left\{\mathbf{C}=\left(C_{i}\right)_{i \in N}\right\}$ from $\mathcal{C}$. Therefore, a bargaining solution in this context is a correspondence $\boldsymbol{F}$ which associates to every $\mathcal{C} \in \Sigma$, a non-empty subset $\boldsymbol{F}(\mathcal{C}) \subseteq \mathcal{C}$.

Given a social evaluation of opportunity sets $R$ satisfying Monotonicity and Representability and its representation $G$, a well-known solution concept in the standard bargaining theory can be applied to the bargaining problems on opportunity assignments, which is defined as follows:

Egalitarian Solution [Xu and Yoshihara (2019a)]: A bargaining solution $\boldsymbol{F}^{E}$ is the egalitarian solution if and only if: for every $\mathcal{C} \in \Sigma, \boldsymbol{F}^{E}(\mathcal{C})=\left\{\mathbf{C}=\left(C_{1}, \cdots, C_{n}\right) \in \mathcal{C} \mid\left(C_{i}, C_{j}\right) \in\right.$ I holds for any $i, j \in N$ and there is no other $\mathbf{C}^{\prime} \in \mathcal{C}$ such that $\left(C_{i}^{\prime}, C_{i}\right) \in P$ for all $\left.i \in N\right\}$.

Thus, the solution $\boldsymbol{F}^{E}$ selects all the undominated assignments, such that in each of these assignments, everyone's opportunity is indifferent with any other's in terms of $R$.

\subsubsection{Axioms on bargaining solutions}

In this subsection, axioms on bargaining solutions over opportunity assignments are presented. The first axiom is the corresponding weak efficiency axiom in standard bargaining models.

Weak Efficiency ${ }^{O B}\left(\mathbf{W E}^{O B}\right)$ : For each $\mathcal{C} \in \Sigma$ and each $\mathbf{C}=\left(C_{i}\right)_{i \in N} \in \boldsymbol{F}(\mathcal{C})$, there is no $\mathbf{C}^{\prime}=\left(C_{i}^{\prime}\right)_{i \in N} \in \mathcal{C}$ such that for every $i \in N, C_{i}^{\prime}>C_{i}$. 
Therefore, the axiom $\left(\mathrm{WE}^{O B}\right)$ requires that the solution should not select an opportunity assignment that is strictly dominated by another feasible opportunity assignment.

To introduce our next axiom, we first define a symmetric problem. We say that $\mathcal{C} \in \Sigma$ is symmetric if for every permutation $\pi: N \rightarrow N$, and for every $\mathbf{C}=\left(C_{i}\right)_{i \in N} \in \mathcal{C}, \pi(\mathbf{C}) \equiv$ $\left(C_{\pi(i)}\right)_{i \in N} \in \mathcal{C}$ holds.

$\operatorname{Symmetry}^{O B}\left(\mathbf{S}^{O B}\right):$ For each $\mathcal{C} \in \Sigma$, if (i) $\mathcal{C}$ is symmetric, and (ii) there exists a $\mathbf{C} \in \mathcal{C}$ such that it is weakly efficient in $\mathcal{C}$ and $C_{i}=C_{j}$ for all $i, j \in N$, then $\mathbf{C} \in \boldsymbol{F}(\mathcal{C})$ holds, and there is no $\mathbf{C}^{\prime} \in \boldsymbol{F}(\mathcal{C})$ such that $C_{i}^{\prime}>C_{j}^{\prime}$ for some $i, j \in N$.

The axiom $\left(\mathrm{S}^{O B}\right)$ stipulates that, for each symmetric problem, if this problem contains a weakly efficient opportunity assignment such that all agents' opportunity sets are identical, then such an opportunity assignment becomes $\boldsymbol{F}$-optimal and no other $\boldsymbol{F}$-optimal opportunity assignment is unequal, in that someone's opportunity set strictly dominates another individual's opportunity set.

The following axiom is analogous to the axiom of contraction independence in standard bargaining models:

Contraction Independence ${ }^{O B}\left(\mathrm{CI}^{O B}\right):$ For each $\mathcal{C}, \mathcal{C}^{\prime} \in \Sigma$ with $\mathcal{C} \supseteq \mathcal{C}^{\prime}$, if $\boldsymbol{F}(\mathcal{C}) \cap \mathcal{C}^{\prime} \neq \varnothing$, then $\boldsymbol{F}\left(\mathcal{C}^{\prime}\right)=\boldsymbol{F}(\mathcal{C}) \cap \mathcal{C}^{\prime}$.

The axiom $\left(\mathrm{CI}^{O B}\right)$ corresponds to Nash's Independence of Irrelevant Alternatives in standard bargaining models.

Our final axiom is an informational requirement on a solution to a problem and is stated below:

Informational Invariance ${ }^{O B}\left(\mathbf{I I}^{O B}\right):$ For each $\mathcal{C} \in \Sigma$ and each $\mathbf{C}=\left(C_{i}\right)_{i \in N}, \mathbf{C}^{\prime}=$ $\left(C_{i}^{\prime}\right)_{i \in N} \in \mathcal{C}$, if $\mathbf{C} \in \boldsymbol{F}(\mathcal{C})$ and $\left(C_{i}^{\prime}, C_{i}\right) \in I$ for all $i \in N$, then $\mathbf{C}^{\prime} \in \boldsymbol{F}(\mathcal{C})$.

According to the axiom ( $\mathrm{II}^{O B}$ ), if two opportunity assignments are "equivalent" in the sense that the two opportunity sets for each and every individual specified by the corresponding opportunity assignments are ranked equally, then whenever one opportunity assignment is $\boldsymbol{F}$-optimal, the other opportunity assignment should be $\boldsymbol{F}$-optimal as well.

\subsubsection{A characterization of the egalitarian solution}

By means of the above four axioms, the egalitarian solution to bargaining problems on opportunity assignments can be characterized:

Theorem 20 [Xu and Yoshihara (2019a)]: The egalitarian solution $\boldsymbol{F}^{E}$ is the unique solution satisfying $\left(\mathrm{WE}^{O B}\right),\left(\mathrm{S}^{O B}\right),\left(\mathrm{CI}^{O B}\right)$ and $\left(\mathrm{II}^{O B}\right)$.

Intuitive idea behind the proof. Instead of giving a proof of this theorem, we discuss the basic intuition about why this theorem holds. ${ }^{9}$

\footnotetext{
${ }^{9}$ For a formal proof of Theorem 20, see Xu and Yoshihara (2019a; Theorem 1).
} 
First, it would be helpful to see that the solution $\boldsymbol{F}^{E}$ is well-defined in the sense that for each $\mathcal{C} \in \Sigma, \boldsymbol{F}^{E}(\mathcal{C})$ is non-empty. Let $G$ be the real-valued, ordinal representation of the social ordering $R$. For each bargaining problem $\mathcal{C} \in \Sigma$, let us define

$$
G(\mathcal{C}) \equiv\left\{G(\mathbf{C})=\left(G\left(C_{i}\right)\right)_{i \in N} \in \mathbb{R}_{+}^{n} \mid \mathbf{C} \in \mathcal{C}\right\}
$$

Let $\partial G(\mathcal{C})$ be the upper boundary of $G(\mathcal{C})$. Since $\mathcal{C}$ is derived from an underlying economic environment $\mathbf{e}=\left(M, \mathbf{u}, \mathbf{c}^{m}, \omega_{M}\right) \in \mathcal{E}$, where $\mathbf{c}^{m}$ is a profile of opportunity correspondences satisfying the requirements (a), (b), (c), and (d), and $G$ is continuous on $\mathcal{K}, \partial G(\mathcal{C})$ constitutes a connected set in $\mathbb{R}_{+}^{n}$. Moreover, since $\mathcal{C}$ is comprehensive ${ }^{10}$ by the requirements (a), (b), and (d) of opportunity correspondences, $G(\mathcal{C})$ must be comprehensive. Finally, by choosing $G(\{\mathbf{0}\})=0$ for the zero vector $\mathbf{0} \in \mathbb{R}_{+}^{k}, G(\mathcal{C})$ has $\mathbf{0} \in \mathbb{R}_{+}^{n}$ as its element, since $\underbrace{(\{\mathbf{0}\}, \ldots,\{\mathbf{0}\})}_{n \text {-times }} \in \mathcal{C}$. Therefore, $G(\mathcal{C})$ corresponds to a standard normalized, non-convex, and comprehensive bargaining problem. Given these observations, we can easily see that the solution $\boldsymbol{F}^{E}$ is well-defined. This is because, for each $\mathcal{C} \in \Sigma$, its corresponding $\partial G(\mathcal{C})$ always contains the vector of equal real numbers $(r, \ldots, r)$, and the inverse image of this vector constitutes the set $\boldsymbol{F}^{E}(\mathcal{C})$. That is, $G^{-1}(r, \ldots, r)=\boldsymbol{F}^{E}(\mathcal{C})$.

Then, if a solution $\boldsymbol{F}$ satisfies $\left(\mathrm{WE}^{O B}\right),\left(\mathrm{S}^{O B}\right),\left(\mathrm{CI}^{O B}\right)$ and $\left(\mathrm{II}^{O B}\right)$, it can be shown that a composite mapping Go $\boldsymbol{F}$ satisfies the axioms (WE), (SS), and (CI) introduced in Section 3 for the standard nonconvex bargaining solutions. Therefore, the map $G \circ \boldsymbol{F}$ selects the weakly efficient and egalitarian utility allocation $(r, \ldots, r) \in \partial G(\mathcal{C})$ for every $\mathcal{C} \in \Sigma$, according to Theorem 16. In conclusion, $G \circ \boldsymbol{F}=G \circ \boldsymbol{F}^{E}$ follows. $\diamond$

\section{Conclusion}

We have reviewed several developments of the recent literature on nonconvex bargaining problems and their applications. From our analysis, it is fair to say that both the KalaiSmorodinsky solution and the egalitarian solution to nonconvex bargaining problems are straightforward extensions of their counterparts in convex bargaining problems due to the single-valuedness of these two solutions and comprehensiveness of bargaining sets. The Nash solution, both symmetric and asymmetric, to nonconvex bargaining problems is, on the other hand, more complex than the Kalai-Smorodinsky and the egalitarian solutions since the Nash solution is in general multi-valued in the context of nonconvex bargaining problems. Faced with a multi-valued Nash solution (a Nash set) to a nonconvex bargaining problem, the issue of narrowing down a 'Nash set' by eliminating some candidates in the Nash set that are deemed to be 'unattractive' may arise. For this purpose, we have touched on one particular selection procedure that tries to incorporate the information about 'equity' aspects of the candidates in a (symmetric) Nash set (see the equitable Nash solution as characterized in Theorem 8): the equity aspect of the equitable Nash solution is concerned about the "normalized dispersion" in a candidate and the invoked procedure is to eliminate those that have larger normalized dispersions than the rest in the Nash set. Admittedly, this

\footnotetext{
${ }^{10} \mathcal{C}$ is comprehensive if, for each $\mathbf{C} \in \mathcal{C}$ and each $i \in N$ with $C_{i} \neq\{\mathbf{0}\}$, there exists $\mathbf{C}^{\prime} \in \mathcal{C}$ such that $C_{i}^{\prime}<C_{i}$ and $C_{j}^{\prime} \subseteq C_{j}$ for all $j \in N \backslash\{i\}$.
} 
is just one possibility and there seem to have other ways of eliminating certain 'unattractive' candidate(s) in a Nash set. It would be interesting to examine the possibilities along this line of thinking. Alternatively, one could go along a path suggested by the work of Peters and Vermeulen (2012) to explicitly impose certain appealing axioms on a solution to narrow down the Nash set and to select some final candidates. Notice that, in this case, one would take the axioms, (WE), (SI) and (CI), as the three basic axioms to be satisfied by a solution in this endeavor. The focus here is not on selecting a candidate from a (symmetric) Nash set. Rather, its focus is on selecting a candidate from an asymmetric Nash set of a nonconvex bargaining problem. This seems another promising line of research.

We have also discussed two applications of nonconvex bargaining theories in the problem of resource allocations. As shown in those two applications, the theories of nonconvex bargaining can be fruitfully applied in those contexts since nonconvex bargaining problems

arise naturally there. It would be interesting to explore many other applications in those contexts and perhaps beyond.

\section{Conflict of interest statement}

On behalf of all authors, the corresponding author states that there is no conflict of interest.

\section{Acknowledgement}

We are grateful to two anonymous referees and the Editor, Walter Trockel, for helpful comments and advice on an earlier version of the paper.

\section{References}

1. Anant,T.C.A., Mukherji, B. and K. Basu (1990): "Bargaining without convexity: generalizing the Kalai-Smorodinsky solution," Economics Letters 33.

2. Arneson, R. (1989): "Equality and equal opportunity for welfare," Philosophical Studies 56, 77-93.

3. Bishop, R. (1960), "Duopoly: Collusion or Warfare?" American Economic Review 50, 933-61.

4. Bossert, W. (1994): "Rational choice and two-person bargaining solutions," Journal of Mathematical Economics 23, 549-563.

5. Chen, M. and E. Maskin (1999): "Bargaining, Production, and Monotonicity in Economic Environments," Journal of Economic Theory 89, 140-147.

6. Chernoff, H. (1954): "Rational selection of decision functions," Econometrica 22, 422443. 
7. Cohen, G.A. (1993): "Equality of what ? On welfare, goods, and capabilities," in The Quality of Life, (edited by Nussbaum, M. and A. Sen), Oxford Univ. Press: Oxford, 9-29.

8. Conley, J. and S. Wilkie (1991): "The bargaining problem without convexity: extending the egalitarian and Kalai-Smorodinsky solutions," Economics Letters 36, 365-369.

9. Conley, J. and S. Wilkie (1996): "An extension of the Nash bargaining solution to nonconvex problems," Games and Economic Behavior 13, 26-38.

10. Denicolò, V. and M. Mariotti (2000): Nash Bargaining Theory, Nonconvex Problems and Social Welfare Orderings, Theory and Decision 48 (4):351-358.

11. Dworkin, R. (1981a): "What is Equality? Part 1: Equality of Welfare," Philosophy and Public Affairs 10, 185-246.

12. Dworkin, R. (1981b): "What is Equality? Part 2: Equality of Resources," Philosophy and Public Affairs 10, 283-345.

13. Fleurbaey, M. and F. Maniquet (1999): "Fair Allocation with Unequal Production Skills: the Solidarity Approach to Compensation," Social Choice and Welfare 16, 569584 .

14. Gaertner, W. and Y. Xu (2006): "Capability as the Basis for a New Measure of the Standard of Living," Journal of Human Development 7(3), 311-321.

15. Ginés, M. and F. Marhuenda (2000): "Welfarism in Economic Domains," Journal of Economic Theory 93, 191-204.

16. Gorman, W. (1980): "The demand for related goods: a possible procedure for analysing quality differentials in the egg market," Review of Economic Studies 47, 843-856.

17. Hammond, P. (1976): "Equity, Arrow's conditions, and Rawls' difference principle," Econometrica 44, 793-804.

18. Herrero, M. J. (1989): "The Nash program: non-convex bargaining problems," Journal of Economic Theory 49, 266-277.

19. Herrero, C. (1996): "Capabilities and utilities," Economic Design 2, 69-88.

20. Kalai, E. (1977): "Proportional solutions to bargaining situations: interpersonal utility comparisons," Econometrica 45, 1623-1630.

21. Kalai, E. and M. Smorodinsky (1975): "Other solutions to Nash's bargaining problem," Econometrica 43, 513-518.

22. Kaneko, M. (1980): "An extension of the Nash bargaining problem and the Nash social welfare function," Theory and Decision 12, 135-148. 
23. Kranich, L. (1996): "Equitable opportunities: An axiomatic approach," Journal of Economic Theory 71, 131-147.

24. Lancaster, K. J., (1966): "A new approach to consumer theory," Journal of Political Economy 74, 132-57.

25. Mariotti, M. (1998): "Nash bargaining theory when the number of alternatives can be finite," Social Choice and Welfare 15, 413-421.

26. Mariotti, M. (1999): "Fair bargains: distributive justice and Nash bargaining theory," Review of Economic Studies 66, 733-741.

27. Mariotti, M. (2000): "Maximal symmetry and the Nash solution," Social Choice and Welfare 17, 45-53

28. Nagahisa, R. and M. Tanaka (2002): "An axiomatization of the Kalai-Smorodinsky solution when the feasible sets can be finite," Social Choice and Welfare 19, 751-761.

29. Nash, J. F. (1950): "The bargaining problem," Econometrica 18, 155-162.

30. Nussbaum, M. (1988): "Nature, function and capability: Aristotle on political distribution," Oxford Studies in Ancient Philosophy, I (supplementary), 145-84.

31. Nussbaum, M. (1993): "Non-relative virtues: an Aristotelian approach", in M. Nussbaum and A.K. Sen (eds.), The Quality of Life, Clarendon Press, Oxford.

32. Nussbaum, M. (2000): Women and Human Development: The Capability Approach, Kali for Women, New Delhi.

33. Pattanaik, P.K. and Y. Xu (2000): "On ranking opportunity sets in economic environments," Journal of Economic Theory 93(1), 48-71.

34. Pattanaik, P.K. and Y. Xu (2007): "Minimal relativism, dominance, and standard of living comparisons based on functionings," Oxford Economic Papers 59(2), 354-374.

35. Peters, H.J.M. and D. Vermeulen (2012): "WPO, COV and IIA bargaining solutions for non-convex bargaining problems," International Journal of Game Theory 41, 851884.

36. Peters, H. and P. Wakker (1991): "Independence of irrelevant alternatives and revealed groups preferences," Econometrica 59, 1787-1801.

37. Qin, C-Z., Tan, G. and A. Wong (2017): Characterization and implementation of Nash bargaining solutions with non-convexity, USC Dornsife Institute for New Economic Thinking Working Paper No. 17-30, University of Southern California.

38. Roemer, J. E. (1986): "Equality of Resources Implies Equality of Welfare," Quarterly Journal of Economics 101, 751-784. 
39. Roemer, J. E. (1986a) "The Mismarriage of Bargaining Theory and Distributive Justice," Ethics 97 (1986), 88-110.

40. Roemer, J. E. (1988): "Axiomatic Bargaining Theory on Economic Environments," Journal of Economic Theory 45, 1-31.

41. Roth, A. (1977a): "Individual Rationality and Nash's Solution to the Bargaining Problem," Mathematics of Operation Research 2, 64-65.

42. Roth, A. (1977b): "Independence of Irrelevant Alternatives, and Solutions to Nash's Bargaining Problem," Journal of Economic Theory 16, 247-251.

43. Samuelson, P.A. (1938): "A note on the pure theory of consumer's behavior," Economica, 61-71.

44. Samuelson, P.A. (1947). Foundations of Economic Analysis (Cambridge, MA: Harvard University Press).

45. Sänchez, M.C. (2000): "Rationality of bargaining solutions," Journal of Mathematical Economics 33, 389-399.

46. Sen, A.K. (1971): Choice functions and revealed preference, Review of Economic Studies 38, 307-317.

47. Sen, A. K. (1980): "Equality of what?" in Tanner Lectures on Human Values. Vol. 1, (edited by McMurrin, S.), Cambridge Univ. Press: Cambridge.

48. Sen, A. K. (1985): Commodities and capabilities, Amsterdam: North-Holland.

49. Sen, A. K. (2002): Rationality and freedom, Harvard University Press: Cambridge, MA.

50. Trockel, W. (1999): Rationalizability of the Nash Bargaining Solution, Journal of Economics Suppl., 159-165.

51. Tsui, K. and J.A. Weymark (1997): Social welfare orderings for ratio-scale measurable utilities, Economic Theory 10(2), 241-256.

52. Xu, Y. (2002): Functioning, capability and the standard of living - an axiomatic approach, Economic Theory 20(2), 387-399.

53. Xu, Y. (2004): "On ranking linear budget sets in terms of freedom of choice," Social Choice and Welfare 22(1), 281-289.

54. Xu, Y. and N. Yoshihara (2006): "Alternative characterizations of three bargaining solutions for nonconvex problems," Games and Economic Behavior 57, 86-92.

55. Xu, Y. and N. Yoshihara (2008): "The Behavior of Solutions to Bargaining Problems on the Basis of Solidarity," Japanese Economic Review 57(1), 86-92. 
56. Xu, Y. and N. Yoshihara (2013): "Rationality and solutions to nonconvex bargaining problems: rationalizability and Nash solutions," Mathematical Social Sciences 66, 6670.

57. Xu, Y. and N. Yoshihara (2019): "An equitable Nash solution to nonconvex bargaining problems," International Journal of Game Theory 48(3), 769-779.

58. Xu, Y. and N. Yoshihara (2019a): "Bargaining theory over opportunity assignments and the egalitarian solution," mimeo.

59. Yoshihara, N. (2003): "Characterizations of bargaining solutions in production economies with unequal skills," Journal of Economic Theory 108, 256-285.

60. Yoshihara, N., (2006): "Solidarity and cooperative bargaining solutions," in A. Wieczorek, M. Malawski, and A. Wiszniewska-Matyszkiel, eds., Game Theory and Mathematical Economics, Banach Center Publications 70, Warszawa, pp. 317-330.

61. Zambrano, E. (2016): "Vintage Nash bargaining without convexity," Economics Letters $141(\mathrm{C}), 32-34$.

62. Zhou, L. (1997): "The Nash bargaining theory with non-convex problems," Econometrica 65, 681-686. 